\title{
Sharp upper bounds for a singular perturbation problem related to micromagnetics
}

\author{
ARKADY POLIAKOVSKY
}

Abstract. We construct an upper bound for the following family of functionals $\left\{E_{\varepsilon}\right\}_{\varepsilon>0}$, which arises in the study of micromagnetics:

$$
E_{\varepsilon}(u)=\int_{\Omega} \varepsilon|\nabla u|^{2}+\frac{1}{\varepsilon} \int_{\mathbb{R}^{2}}\left|H_{u}\right|^{2} .
$$

Here $\Omega$ is a bounded domain in $\mathbb{R}^{2}, u \in H^{1}\left(\Omega, S^{1}\right)$ (corresponding to the magnetization) and $H_{u}$, the demagnetizing field created by $u$, is given by

$$
\begin{cases}\operatorname{div}\left(\tilde{u}+H_{u}\right)=0 & \text { in } \mathbb{R}^{2}, \\ \operatorname{curl} H_{u}=0 & \text { in } \mathbb{R}^{2},\end{cases}
$$

where $\tilde{u}$ is the extension of $u$ by 0 in $\mathbb{R}^{2} \backslash \Omega$. Our upper bound coincides with the lower bound obtained by Rivière and Serfaty.

Mathematics Subject Classification (2000): 49J45 (primary); 35B25, 35J20 (secondary).

\section{Introduction}

In this paper we study the following energy-functional, related to micromagnetics:

$$
E_{\varepsilon}(u):=\int_{\Omega} \varepsilon|\nabla u|^{2}+\frac{1}{\varepsilon} \int_{\mathbb{R}^{2}}\left|H_{u}\right|^{2} .
$$

Here $\Omega$ is a bounded domain in $\mathbb{R}^{2}$ with Lipschitz boundary, $u$ is a unit-valued vector-field (corresponding to the magnetization) in $H^{1}\left(\Omega, S^{1}\right)$ and $H_{u}$, the demagnetizing field created by $u$, is given by

$$
\left\{\begin{array}{lc}
\operatorname{div}\left(\tilde{u}+H_{u}\right)=0 & \text { in } \mathbb{R}^{2} \\
\operatorname{curl} H_{u}=0 & \text { in } \mathbb{R}^{2},
\end{array}\right.
$$

Received December 5, 2006; accepted in revised form November 22, 2007. 
where $\tilde{u}$ is the extension of $u$ by 0 in $\mathbb{R}^{2} \backslash \Omega$. For the physical models related to $E_{\varepsilon}$, we refer to [18] and all the references therein.

We can rewrite (1.1) in the following form. Denoting by $\Delta^{-1} \tilde{u}$ the Newtonian potential of $\tilde{u}$, we observe that the vector-field $\bar{H}_{u}:=-\nabla\left(\operatorname{div}\left(\Delta^{-1} \tilde{u}\right)\right)$ belongs to $L^{2}\left(\mathbb{R}^{2}, \mathbb{R}^{2}\right)$. Moreover,

$$
\begin{cases}\operatorname{div} \bar{H}_{u}=-\operatorname{div} \tilde{u} & \text { in } \mathbb{R}^{2} \\ \operatorname{curl} \bar{H}_{u}=0 & \text { in } \mathbb{R}^{2}\end{cases}
$$

So $H_{u}=\bar{H}_{u}$ and we obtain

$$
E_{\varepsilon}(u)=\int_{\Omega} \varepsilon|\nabla u|^{2}+\frac{1}{\varepsilon} \int_{\mathbb{R}^{2}}\left|\nabla\left(\operatorname{div}\left(\Delta^{-1} \tilde{u}\right)\right)\right|^{2} .
$$

In [19] T. Rivière and S. Serfaty proved the following theorem, giving compactness and a lower bound for the energies $E_{\varepsilon}$.

Theorem 1.1. Let $\Omega$ be a bounded simply connected domain in $\mathbb{R}^{2}$. Let $\varepsilon_{n} \rightarrow 0$ and $u_{n} \in H^{1}\left(\Omega, S^{1}\right)$ with a lifting $\varphi_{n} \in H^{1}(\Omega, \mathbb{R})$ i.e., $u_{n}=e^{i \varphi_{n}}$ a.e., and such that

$$
\begin{gathered}
E_{\varepsilon_{n}}\left(u_{n}\right) \leq C \\
\left\|\varphi_{n}\right\|_{L^{\infty}(\Omega)} \leq N .
\end{gathered}
$$

Then, up to extraction of a subsequence, there exists $u$ and $\varphi$ in $\cap_{q<\infty} L^{q}(\Omega)$ such that

$$
\begin{aligned}
& \varphi_{n} \rightarrow \varphi \text { in } \cap_{q<\infty} L^{q}(\Omega) \\
& u_{n} \rightarrow u \text { in } \cap_{q<\infty} L^{q}(\Omega) .
\end{aligned}
$$

Moreover, if we consider

$$
\left\{\begin{array}{l}
T^{t} \varphi(x):=\inf (\varphi(x), t) \\
T^{t} u(x):=e^{i T^{t} \varphi(x)}
\end{array}\right.
$$

then $\operatorname{div}_{x} T^{t} u$ is a bounded Radon measure on $\Omega \times \mathbb{R}$, with $t \mapsto \operatorname{div}_{x} T^{t} u$ continuous from $\mathbb{R}$ to $\mathcal{D}^{\prime}(\Omega)$. In addition

$$
2 \int_{\mathbb{R}} \int_{\Omega}\left|\operatorname{div}_{x} T^{t} u\right| d x d t \leq \underline{\lim _{n \rightarrow \infty}} \int_{\Omega} 2\left|\nabla \varphi_{n} \cdot H_{u_{n}}\right| \leq \underset{n \rightarrow \infty}{\lim _{n \rightarrow \infty}} E_{\varepsilon_{n}}\left(u_{n}\right)<\infty .
$$

The main contribution of this paper is to establish the upper bound for $E_{\varepsilon}$ in the case where $u$ and its lifting $\varphi$ belong to $B V$. First of all we observe that if $\varepsilon_{n} \rightarrow 0$, 
$E_{\varepsilon_{n}}\left(u_{n}\right) \leq C$, and $u_{n} \rightarrow u$ in $L^{q}$, where $\left|u_{n}\right|=1$ and $u \in B V$ then clearly $\lim _{n \rightarrow \infty} \int_{\mathbb{R}^{2}}\left|H_{u_{n}}\right|^{2}=0$, which implies

$$
\lim _{n \rightarrow \infty} \int_{\mathbb{R}^{2}} \tilde{u}_{n} \cdot \nabla \delta=-\lim _{n \rightarrow \infty} \int_{\mathbb{R}^{2}} H_{u_{n}} \cdot \nabla \delta=0 \quad \forall \delta \in C_{c}^{\infty}\left(\mathbb{R}^{2}, \mathbb{R}\right) .
$$

Therefore, $\operatorname{div} \tilde{u}=0$ as a distribution, i.e.,

$$
\begin{cases}|u|=1 & \text { a.e. in } \Omega \\ \operatorname{div} u=0 & \text { in } \Omega \\ u \cdot \boldsymbol{n}=0 & \text { on } \partial \Omega\end{cases}
$$

The main result of this paper is the following theorem.

Theorem 1.2. Let $\Omega$ be a bounded domain in $\mathbb{R}^{2}$ with Lipschitz boundary. Consider $u \in B V\left(\Omega, S^{1}\right)$, satisfying $\operatorname{div} u=0$ in $\Omega$ and $u \cdot \boldsymbol{n}=0$ on $\partial \Omega$ and assume there exist $\varphi \in B V(\Omega, \mathbb{R})$, such that $u=e^{i \varphi}$ a e. in $\Omega$. Then there exists a family of functions $\left\{v_{\varepsilon}\right\} \subset C^{2}\left(\mathbb{R}^{N}, \mathbb{R}\right)$ satisfying

$$
\lim _{\varepsilon \rightarrow 0^{+}} v_{\varepsilon}(x)=\varphi(x) \quad \text { in } \quad L^{1}(\Omega, \mathbb{R})
$$

and

$$
\lim _{\varepsilon \rightarrow 0} E_{\varepsilon}\left(e^{i v_{\varepsilon}}\right)=2 \int_{\mathbb{R}} \int_{\Omega}\left|\operatorname{div}_{x} T^{t} u\right| d x d t .
$$

Moreover, if $\varphi \in B V(\Omega, \mathbb{R}) \cap L^{\infty}$, then we have

$$
\lim _{\varepsilon \rightarrow 0^{+}} v_{\varepsilon}(x)=\varphi(x) \quad \text { in } \quad L^{p}(\Omega, \mathbb{R}) \quad \forall p \in[1, \infty) .
$$

In order to construct $\left\{v_{\varepsilon}\right\}$ we take the convolution of $\varphi$ with a varying smoothing kernel, i.e., we set $v_{\varepsilon}(x):=\varepsilon^{-2} \int_{\mathbb{R}^{2}} \eta\left(\frac{y-x}{\varepsilon}, x\right) \varphi(y) d y$ where $\eta \in C_{c}^{2}\left(\mathbb{R}^{2} \times \mathbb{R}^{2}\right)$ satisfies $\int_{\mathbb{R}^{2}} \eta(z, x) d x=1$ for every $x \in \Omega$, and we optimize the choice of the kernel $\eta$. A similar approach was used in [16] and [17], but a new ingredient is required here, since the non-local term $\int_{\mathbb{R}^{2}}\left|H_{u}\right|^{2}$ gives certain difficulties.

\subsection{The basic idea}

We shall follow essentially the strategy of [16] and [17]. The main new ingredient here is the calculation of

$$
\lim _{\varepsilon \rightarrow 0^{+}} \frac{1}{\varepsilon} \int_{\mathbb{R}^{2}}\left|\nabla\left(\operatorname{div}\left(\Delta^{-1}\left\{\chi_{\Omega} e^{i v_{\varepsilon}}\right\}\right)\right)\right|^{2} .
$$

We first calculate

$$
L(l):=\lim _{\varepsilon \rightarrow 0^{+}} \frac{1}{\varepsilon} \int_{\mathbb{R}^{2}}\left|\nabla\left(\operatorname{div}\left(\Delta^{-1}\left\{\varphi_{\varepsilon}\right\}\right)\right)\right|^{2},
$$


where $\varphi_{\varepsilon}(x):=\varepsilon^{-2} \int_{\mathbb{R}^{2}} l\left(\frac{y-x}{\varepsilon}, x\right) \varphi(y) d y$, with $l \in C_{c}^{2}\left(\mathbb{R}^{2} \times \Omega, \mathbb{R}^{2}\right)$ satisfying $\int_{\mathbb{R}^{2}} l(z, x) d x=0$ for every $x \in \Omega$. Since $\nabla\left(\operatorname{div}\left(\Delta^{-1}\left\{\phi_{\varepsilon}\right\}\right)\right)$ has the same asymptotic behavior as $\varepsilon^{-2} \int_{\mathbb{R}^{2}} s\left(\frac{y-x}{\varepsilon}, x\right) \varphi(y) d y$, where $s(z, x):=\nabla_{z}\left(\operatorname{div}_{z}\left(\Delta_{z}^{-1} l(z, x)\right)\right)$, we can calculate the limit $L(l)$ in a similar way to what was done in [16] and [17] (see Lemmas 3.1 and 3.2 for the details). Using the results of [17] (see Proposition 2.2 below) it is easy to calculate

$$
D(l):=\lim _{\varepsilon \rightarrow 0} \frac{1}{\varepsilon} \int_{\Omega}\left|e^{i v_{\varepsilon}}-\varphi_{\varepsilon}-u\right|^{2} d x .
$$

Now, given a fixed $\eta$ and a small $\delta>0$, we choose $l=l_{\delta}$ in such a way that $D\left(l_{\delta}\right)<\delta$. Then, using the estimate

$$
\int_{\mathbb{R}^{2}}\left|\nabla\left(\operatorname{div}\left(\Delta^{-1}\left\{\chi_{\Omega} f\right\}\right)\right)\right|^{2} \leq C \int_{\Omega}|f|^{2},
$$

we deduce that

$$
\begin{aligned}
& \left.\varlimsup_{\varepsilon \rightarrow 0}\left|\frac{1}{\varepsilon} \int_{\mathbb{R}^{2}}\right| \nabla\left(\operatorname{div}\left(\Delta^{-1}\left\{\chi_{\Omega} e^{i v_{\varepsilon}}\right\}\right)\right)\right|^{2}-\frac{1}{\varepsilon} \int_{\mathbb{R}^{2}}\left|\nabla\left(\operatorname{div}\left(\Delta^{-1}\left\{\chi_{\Omega} \varphi_{\varepsilon}\right\}\right)\right)\right|^{2} \mid \\
& \leq \varlimsup_{\varepsilon \rightarrow 0} C\left\{\frac{1}{\varepsilon} \int_{\mathbb{R}^{2}}\left|\nabla\left(\operatorname{div}\left(\Delta^{-1}\left\{\chi_{\Omega}\left(e^{i v_{\varepsilon}}-\varphi_{\varepsilon}-u\right)\right\}\right)\right)\right|^{2}\right\}^{1 / 2} \leq C \delta^{1 / 2} .
\end{aligned}
$$

Finally, tetting $\delta$ tend to 0 , we conclude that the limit in (1.7) should be equal to $\lim _{\delta \rightarrow 0} L\left(l_{\delta}\right)$. We follow basically this strategy in the proof of Proposition 4.1.

ACKnOwledgements. I am grateful to Prof. Camillo De Lellis for proposing this problem to me and for some useful suggestions. Part of this research was done during a visit at the Laboratoire J. L. Lions of the University Paris VI in the framework of the RTN-Programme Fronts-Singularities. I am indebted to Prof. Haim Brezis for the invitation and for many stimulating discussions.

\section{Preliminaries}

Throughout this section we assume that $\Omega$ is a bounded domain in $\mathbb{R}^{2}$ with Lipschitz boundary. We begin by introducing some notation. For every $v \in S^{1}$ (the unit sphere in $\mathbb{R}^{2}$ ) and $R>0$ we denote

$$
\begin{aligned}
& B_{R}^{+}(x, \boldsymbol{v})=\left\{y \in \mathbb{R}^{2}:|y-x|<R,(y-x) \cdot \boldsymbol{v}>0\right\}, \\
& B_{R}^{-}(x, \boldsymbol{v})=\left\{y \in \mathbb{R}^{2}:|y-x|<R,(y-x) \cdot \boldsymbol{v}<0\right\}, \\
& H_{+}(x, \boldsymbol{v})=\left\{y \in \mathbb{R}^{2}:(y-x) \cdot \boldsymbol{v}>0\right\}, \\
& H_{-}(x, \boldsymbol{v})=\left\{y \in \mathbb{R}^{2}:(y-x) \cdot \boldsymbol{v}<0\right\}
\end{aligned}
$$


and

$$
H_{v}^{0}=\left\{y \in \mathbb{R}^{2}: y \cdot v=0\right\}
$$

Definition 2.1. Consider a function $f \in B V\left(\Omega, \mathbb{R}^{m}\right)$ and a point $x \in \Omega$.

i) We say that $x$ is a point of approximate continuity of $f$ if there exists $z \in \mathbb{R}^{m}$ such that

$$
\lim _{\rho \rightarrow 0^{+}} \frac{\int_{B_{\rho}(x)}|f(y)-z| d y}{\mathcal{L}^{2}\left(B_{\rho}(x)\right)}=0 .
$$

In this case $z$ is called an approximate limit of $f$ at $x$ and we denote $z$ by $\tilde{f}(x)$. The set of points of approximate continuity of $f$ is denoted by $G_{f}$.

ii) We say that $x$ is an approximate jump point of $f$ if there exist $a, b \in \mathbb{R}^{m}$ and $v \in S^{N-1}$ such that $a \neq b$ and

$$
\lim _{\rho \rightarrow 0^{+}} \frac{\int_{B_{\rho}^{+}(x, v)}|f(y)-a| d y}{\mathcal{L}^{2}\left(B_{\rho}(x)\right)}=0, \quad \lim _{\rho \rightarrow 0^{+}} \frac{\int_{B_{\rho}^{-}(x, v)}|f(y)-b| d y}{\mathcal{L}^{2}\left(B_{\rho}(x)\right)}=0 .
$$

The triple $(a, b, v)$, uniquely determined by (2.6) up to a permutation of $(a, b)$ and a change of sign of $\boldsymbol{v}$, is denoted by $\left(f^{+}(x), f^{-}(x), \boldsymbol{v}_{f}(x)\right)$. We shall call $\boldsymbol{v}_{f}(x)$ the approximate jump vector and we shall sometimes write simply $\boldsymbol{v}(x)$ if the reference to the function $f$ is clear. The set of approximate jump points is denoted by $J_{f}$. A choice of $\boldsymbol{v}(x)$ for every $x \in J_{f}$ (which is unique up to sign) determines an orientation of $J_{f}$. At a point of approximate continuity $x$, we shall use the convention $f^{+}(x)=f^{-}(x)=\tilde{f}(x)$.

We refer to [2] for the results on BV-functions that we shall use in the sequel.

Consider a function $\Phi=\left(\varphi_{1}, \varphi_{2}, \ldots, \varphi_{d}\right) \in B V\left(\Omega, \mathbb{R}^{d}\right)$. By [2, Proposition 3.21] we may extend $\Phi$ to a function $\bar{\Phi} \in B V\left(\mathbb{R}^{2}, \mathbb{R}^{d}\right)$, such that $\bar{\Phi}=\Phi$ a.e. in $\Omega$, supp $\bar{\Phi}$ is compact and $\|D \bar{\Phi}\|(\partial \Omega)=0$. From the proof of Proposition 3.21 in [2] it follows that if $\Phi \in B V\left(\Omega, \mathbb{R}^{d}\right) \cap L^{\infty}$ then its extension $\bar{\Phi}$ is also in $B V\left(\mathbb{R}^{2}, \mathbb{R}^{d}\right) \cap L^{\infty}$. Consider also a matrix valued function $\Xi \in C_{c}^{2}\left(\mathbb{R}^{2} \times \mathbb{R}^{2}, \mathbb{R}^{l \times d}\right)$ For every $\varepsilon>0$ define a function $\Psi_{\varepsilon}(x): \mathbb{R}^{2} \rightarrow \mathbb{R}^{l}$ by

$$
\begin{aligned}
\Psi_{\varepsilon}(x) & :=\frac{1}{\varepsilon^{2}} \int_{\mathbb{R}^{2}} \Xi\left(\frac{y-x}{\varepsilon}, x\right) \cdot \bar{\Phi}(y) d y \\
& =\int_{\mathbb{R}^{2}} \Xi(z, x) \cdot \bar{\Phi}(x+\varepsilon z) d z, \quad \forall x \in \mathbb{R}^{2} .
\end{aligned}
$$

Thanks to [17, Proposition 3.2], we have the following result. It generalizes Proposition 3.2 from [16] and provides the key tool for the calculation of the upper bound, both in [17] and in the current paper. In the proof of Lemma 3.2 we shall also follow the general strategy of its proof in [17]. 
Proposition 2.2. Let $W \in C^{1}\left(\mathbb{R}^{l} \times \mathbb{R}^{q}, \mathbb{R}\right)$ satisfying

$$
\nabla_{a} W(a, b)=0 \text { whenever } W(a, b)=0 .
$$

Consider $\Phi \in B V\left(\Omega, \mathbb{R}^{d}\right) \cap L^{\infty}$ and $u \in B V\left(\Omega, \mathbb{R}^{q}\right) \cap L^{\infty}$ satisfying

$$
W\left(\left\{\int_{\mathbb{R}^{2}} \Xi(z, x) d z\right\} \cdot \Phi(x), u(x)\right)=0 \quad \text { for a.e. } x \in \Omega,
$$

where $\Xi \in C_{c}^{2}\left(\mathbb{R}^{2} \times \mathbb{R}^{2}, \mathbb{R}^{l \times d}\right)$, as above. Let $\Psi_{\varepsilon}$ be as in (2.7). Then,

$$
\begin{aligned}
& \lim _{\varepsilon \rightarrow 0} \int_{\Omega} \frac{1}{\varepsilon} W\left(\Psi_{\varepsilon}(x), u(x)\right) d x \\
& =\int_{J_{\Phi}}\left\{\int_{-\infty}^{0} W\left(\Gamma(t, x), u^{+}(x)\right) d t+\int_{0}^{+\infty} W\left(\Gamma(t, x), u^{-}(x)\right) d t\right\} d \mathcal{H}^{1}(x),
\end{aligned}
$$

where

$$
\Gamma(t, x)=\left(\int_{-\infty}^{t} P(s, x) d s\right) \cdot \Phi^{-}(x)+\left(\int_{t}^{+\infty} P(s, x) d s\right) \cdot \Phi^{+}(x),
$$

with

$$
P(t, x)=\int_{H_{v(x)}^{0}} \Xi(t \boldsymbol{v}(x)+y, x) d \mathcal{H}^{1}(y),
$$

$\boldsymbol{v}(x)$ is the jump vector of $\Phi$ and it is assumed that the orientation of $J_{u}$ coincides with the orientation of $J_{\Phi} \mathcal{H}^{1}$ a.e. on $J_{u} \cap J_{\Phi}$.

Definition 2.3. Given $f \in L^{\infty}\left(\mathbb{R}^{2}, \mathbb{R}^{k}\right)$ with compact support, we define its Newtonian potential

$$
\left(\Delta^{-1} f\right)(x):=\int_{\mathbb{R}^{2}} \frac{1}{2 \pi} \ln |x-y| f(y) d y .
$$

Than it is well known that

$$
\int_{\mathbb{R}^{2}}\left|\nabla^{2}\left(\Delta^{-1} f\right)(x)\right|^{2} d x=\int_{\mathbb{R}^{2}}|f(x)|^{2} d x,
$$

where given $v=\left(v_{1}, \ldots, v_{k}\right): \mathbb{R}^{2} \rightarrow \mathbb{R}^{k}$ we denote by $\nabla^{2} v \in \mathbb{R}^{k \times 2 \times 2}$ the tensor with $l i j$-th component $\partial_{i j}^{2} v_{l}$.

Definition 2.4. Let $\mathcal{V}$ be the class of all functions $\eta \in C_{c}^{2}\left(\mathbb{R}^{2} \times \mathbb{R}^{2}, \mathbb{R}\right)$ such that

$$
\int_{\mathbb{R}^{2}} \eta(z, x) d z=1 \quad \forall x \in \Omega .
$$

Let $\mathcal{U}$ be the class of all functions $l(z, x) \in C_{c}^{2}\left(\mathbb{R}^{2} \times \Omega, \mathbb{R}^{2}\right)$ such that

$$
\int_{\mathbb{R}^{2}} l(z, x) d z=0 \quad \forall x \in \mathbb{R}^{2} .
$$


In [17, Lemma 5.1], we proved the following statement. This statement generalize Claim 3 of Lemma 3.4 from [16] and was an essential tool in the optimizing the upper bound in [17].

Lemma 2.5. Let $\mu$ be positive finite Borel measure on $\Omega$ and $\boldsymbol{v}_{0}(x): \Omega \rightarrow \mathbb{R}^{2}$ a Borel measurable function with $\left|\boldsymbol{v}_{0}\right|=1$. Let $\mathcal{W}_{1}$ denote the set of functions $p(t, x): \mathbb{R} \times \Omega \rightarrow \mathbb{R}$ satisfying the following conditions:

i) $p$ is Borel measurable and bounded,

ii) there exists $M>0$ such that $p(t, x)=0$ for $|t|>M$ and any $x \in \Omega$,

iii) $\int_{\mathbb{R}} p(t, x) d t=1, \forall x \in \Omega$.

Then for every $p(t, x) \in \mathcal{W}_{1}$, there exists a sequence of functions $\left\{\eta_{n}\right\} \subset \mathcal{V}$ (see Definition 2.4), such that the sequence of functions $\left\{p_{n}(t, x)\right\}$ defined on $\mathbb{R} \times \Omega$ by

$$
p_{n}(t, x)=\int_{H_{v_{0}(x)}^{0}} \eta_{n}\left(t \boldsymbol{v}_{0}(x)+y, x\right) d \mathcal{H}^{1}(y),
$$

has the following properties:

i) there exists $C_{0}$ such that $\left\|p_{n}\right\|_{L^{\infty}} \leq C_{0}$ for every $n$,

ii) there exist $M>0$ such that $p_{n}(t, \bar{x})=0$ for $|t|>M$ and every $x \in \Omega$, for all $n$.

iii) $\lim _{n \rightarrow \infty} \int_{\Omega} \int_{\mathbb{R}}\left|p_{n}(t, x)-p(t, x)\right| d t d \mu(x)=0$.

With the same method it is not difficult to prove

Lemma 2.6. Let $\mu$ be positive finite Borel measure on $\Omega$ and $\boldsymbol{v}_{0}(x): \Omega \rightarrow \mathbb{R}^{2}$ a Borel measurable function with $\left|\boldsymbol{v}_{0}\right|=1$. Let $\mathcal{W}_{0}$ denote the set of functions $q(t, x): \mathbb{R} \times \Omega \rightarrow \mathbb{R}^{2}$ satisfying the following conditions:

i) $q$ is Borel measurable and bounded,

ii) there exists $M>0$ such that $q(t, x)=0$ for $|t|>M$ and every $x \in \Omega$.

iii) $\int_{\mathbb{R}} q(t, x) d t=0, \forall x \in \Omega$.

Then for every $q(t, x) \in \mathcal{W}_{0}$, there exists a sequence of functions $\left\{l_{n}\right\} \subset \mathcal{U}$ (see Definition 2.4), such that the sequence of functions $\left\{q_{n}(t, x)\right\}$ defined on $\mathbb{R} \times \Omega$ by

$$
q_{n}(t, x)=\int_{H_{v_{0}(x)}^{0}} l_{n}\left(t \boldsymbol{v}_{0}(x)+y, x\right) d \mathcal{H}^{1}(y),
$$

has the following properties:

i) there exists $C_{0}$ such that $\left\|q_{n}\right\|_{L^{\infty}} \leq C_{0}$ for every $n$,

ii) there exist $M>0$ such that $q_{n}(t, x)=0$ for $|t|>M$ and every $x \in \Omega$, for all $n$,

iii) $\lim _{n \rightarrow \infty} \int_{\Omega} \int_{\mathbb{R}}\left|q_{n}(t, x)-q(t, x)\right| d t d \mu(x)=0$. 


\section{First estimates}

Throughout this section we assume that $\Omega$ is a bounded domain in $\mathbb{R}^{2}$ with Lipschitz boundary.

Let $l \in \mathcal{U}$ (see Definition 2.4). Consider $r(z, x):=\Delta_{z}^{-1} l(z, x)$. Then $r \in$ $C^{2}\left(\mathbb{R}^{2} \times \mathbb{R}^{2}, \mathbb{R}^{2}\right)$ with supp $r \subset \mathbb{R}^{2} \times K$, where $K \Subset \Omega$. Moreover, since $\int_{\mathbb{R}^{2}} l(z, x) d z=0$, for every $k=0,1,2 \ldots$ we have the estimates

$$
\begin{aligned}
\left|\nabla_{x}^{k} r(z, x)\right| & \leq \frac{C_{k}}{|z|+1}, \\
\left|\nabla_{x}^{k}\left(\nabla_{z} r(z, x)\right)\right| & \leq \frac{C_{k}}{|z|^{2}+1}, \\
\left|\nabla_{x}^{k}\left(\nabla_{z}^{2} r(z, x)\right)\right| & \leq \frac{C_{k}}{|z|^{3}+1},
\end{aligned}
$$

where $C_{k}>0$ does not depend on $z$ and $x$.

Lemma 3.1. Let $\varphi \in B V(\Omega, \mathbb{R}) \cap L^{\infty}$ and $l \in \mathcal{U}$ (see Definition 2.4). For every $\varepsilon>0$ consider the function $\varphi_{\varepsilon} \in C^{1}\left(\mathbb{R}^{2}, \mathbb{R}^{2}\right)$ by

$$
\varphi_{\varepsilon}(x):=\frac{1}{\varepsilon^{2}} \int_{\mathbb{R}^{2}} l\left(\frac{y-x}{\varepsilon}, x\right) \bar{\varphi}(y) d y=\int_{\mathbb{R}^{2}} l(z, x) \bar{\varphi}(x+\varepsilon z) d z,
$$

where $\bar{\varphi}$ is some bounded $B V$ extension of $\varphi$ to $\mathbb{R}^{2}$ with compact support. Next consider $r(z, x):=\Delta_{z}^{-1} l(z, x)$ and set

$$
\begin{aligned}
\xi_{\varepsilon}(x) & :=\frac{1}{\varepsilon^{2}} \int_{\mathbb{R}^{2}} \nabla_{1}\left(\operatorname{div}_{1} r\right)\left(\frac{y-x}{\varepsilon}, x\right) \bar{\varphi}(y) d y \\
& =\int_{\mathbb{R}^{2}} \nabla_{z}\left(\operatorname{div}_{z} r(z, x)\right) \bar{\varphi}(x+\varepsilon z) d z,
\end{aligned}
$$

where $\nabla_{1}\left(\operatorname{div}_{1} r\right)$ is the gradient of divergence of $r(z, x)$ in its first variable, namely z. Then,

$$
\int_{\mathbb{R}^{2}} \frac{1}{\varepsilon}\left|\nabla\left(\operatorname{div}\left(\Delta^{-1} \varphi_{\varepsilon}\right)\right)(x)\right|^{2} d x=o_{\varepsilon}(1)+\int_{\Omega} \frac{1}{\varepsilon} \varphi_{\varepsilon}(x) \cdot \xi_{\varepsilon}(x) d x .
$$

Proof. Since $l(z, x)=0$ if $x \notin K$, where $K$ is some compact subset of $\Omega$, we have, in particular, $\varphi_{\varepsilon}(x)=0$ for every $x \in \mathbb{R}^{2} \backslash \Omega$. Then, integrating by part two times, we conclude

$$
\begin{aligned}
& \int_{\mathbb{R}^{2}} \frac{1}{\varepsilon}\left|\nabla\left(\operatorname{div}\left(\Delta^{-1} \varphi_{\varepsilon}\right)\right)(x)\right|^{2} d x \\
& =-\int_{\mathbb{R}^{2}} \frac{1}{\varepsilon} \Delta\left(\operatorname{div}\left(\Delta^{-1} \varphi_{\varepsilon}\right)\right)(x) \cdot\left(\operatorname{div}\left(\Delta^{-1} \varphi_{\varepsilon}\right)\right)(x) d x \\
& =-\int_{\mathbb{R}^{2}} \frac{1}{\varepsilon} \operatorname{div} \varphi_{\varepsilon}(x) \cdot\left(\operatorname{div}\left(\Delta^{-1} \varphi_{\varepsilon}\right)\right)(x) d x \\
& =\int_{\Omega} \frac{1}{\varepsilon} \varphi_{\varepsilon}(x) \cdot \nabla\left(\operatorname{div}\left(\Delta^{-1} \varphi_{\varepsilon}\right)\right)(x) d x .
\end{aligned}
$$


Next consider the function $\zeta_{\varepsilon} \in C^{1}\left(\mathbb{R}^{2}, \mathbb{R}^{2}\right)$ given by

$$
\zeta_{\varepsilon}(x):=\frac{1}{\varepsilon^{2}} \int_{\mathbb{R}^{2}} r\left(\frac{y-x}{\varepsilon}, x\right) \bar{\varphi}(y) d y=\int_{\mathbb{R}^{2}} r(z, x) \bar{\varphi}(x+\varepsilon z) d z .
$$

We will prove now that

$$
\left|\varepsilon^{2} \nabla^{2} \zeta_{\varepsilon}(x)-\int_{\mathbb{R}^{2}} \nabla_{z}^{2} r(z, x) \bar{\varphi}(x+\varepsilon z) d z\right| \leq C \varepsilon^{2 / 3} \quad \forall x \in \Omega .
$$

We shall denote by $\nabla_{1} l$ and $\nabla_{2} l$ the gradient of $l(z, x)$ with respect to the variables $z$ and $x$ respectively. We have,

$$
\begin{aligned}
& \varepsilon^{2} \nabla^{2} \zeta_{\varepsilon}(x)-\int_{\mathbb{R}^{2}} \nabla_{z}^{2} r(z, x) \bar{\varphi}(x+\varepsilon z) d z \\
& =\int_{\mathbb{R}^{2}} \nabla_{x}^{2} r\left(\frac{y-x}{\varepsilon}, x\right) \bar{\varphi}(y) d y-\frac{1}{\varepsilon^{2}} \int_{\mathbb{R}^{2}} \nabla_{1}^{2} r\left(\frac{y-x}{\varepsilon}, x\right) \bar{\varphi}(y) d y \\
& =-\frac{1}{\varepsilon} \int_{\mathbb{R}^{2}}\left\{\nabla_{1} \nabla_{2} r\left(\frac{y-x}{\varepsilon}, x\right)+\nabla_{2} \nabla_{1} r\left(\frac{y-x}{\varepsilon}, x\right)\right\} \bar{\varphi}(y) d y \\
& \quad+\int_{\mathbb{R}^{2}} \nabla_{2}^{2} r\left(\frac{y-x}{\varepsilon}, x\right) \bar{\varphi}(y) d y .
\end{aligned}
$$

Therefore, by the Hölder inequality and the estimates in (3.1), we obtain

$$
\begin{aligned}
& \left|\varepsilon^{2} \nabla^{2} \zeta_{\varepsilon}(x)-\int_{\mathbb{R}^{2}} \nabla_{z}^{2} r(z, x) \bar{\varphi}(x+\varepsilon z) d z\right| \\
& \leq \varepsilon^{2 / 3}\left(\frac{1}{\varepsilon^{2}} \int_{\mathbb{R}^{2}}\left|\nabla_{1} \nabla_{2} r\left(\frac{y-x}{\varepsilon}, x\right)+\nabla_{2} \nabla_{1} r\left(\frac{y-x}{\varepsilon}, x\right)\right|^{6 / 5} d y\right)^{5 / 6}\left(\int_{\mathbb{R}^{2}}|\bar{\varphi}(y)|^{6} d y\right)^{1 / 6} \\
& \quad+\varepsilon^{2 / 3}\left(\frac{1}{\varepsilon^{2}} \int_{\mathbb{R}^{2}}\left|\nabla_{2}^{2} r\left(\frac{y-x}{\varepsilon}, x\right)\right|^{3} d y\right)^{1 / 3}\left(\int_{\mathbb{R}^{2}}|\bar{\varphi}(y)|^{3 / 2} d y\right)^{2 / 3} \\
& =\varepsilon^{2 / 3}\left(\int_{\mathbb{R}^{2}}\left|\nabla_{1} \nabla_{2} r(z, x)+\nabla_{2} \nabla_{1} r(z, x)\right|^{6 / 5} d z\right)^{5 / 6}\left(\int_{\mathbb{R}^{2}}|\bar{\varphi}(y)|^{6} d y\right)^{1 / 6} \\
& \quad+\varepsilon^{2 / 3}\left(\int_{\mathbb{R}^{2}}\left|\nabla_{2}^{2} r(z, x)\right|^{3} d z\right)^{1 / 3}\left(\int_{\mathbb{R}^{2}}|\bar{\varphi}(y)|^{3 / 2} d y\right)^{2 / 3} \leq C \varepsilon^{2 / 3}
\end{aligned}
$$

which gives (3.7). In particular,

$$
\begin{aligned}
\left|\varepsilon^{2} \Delta \zeta_{\varepsilon}(x)-\varphi_{\varepsilon}(x)\right| & =\left|\varepsilon^{2} \Delta \zeta_{\varepsilon}(x)-\int_{\mathbb{R}^{2}} \Delta_{z} r(z, x) \bar{\varphi}(x+\varepsilon z) d z\right| \\
& \leq C_{0} \varepsilon^{2 / 3}
\end{aligned}
$$


Next by (3.5),

$$
\begin{aligned}
\int_{\mathbb{R}^{2}} \frac{1}{\varepsilon}\left|\nabla\left(\operatorname{div}\left(\Delta^{-1} \varphi_{\varepsilon}\right)\right)(x)\right|^{2} d x= & \int_{\Omega} \frac{1}{\varepsilon} \varphi_{\varepsilon}(x) \cdot \nabla\left(\operatorname{div}\left(\Delta^{-1} \varphi_{\varepsilon}\right)\right)(x) d x \\
= & \int_{\Omega} \frac{1}{\varepsilon} \varphi_{\varepsilon}(x) \cdot \nabla\left(\operatorname{div}\left(\varepsilon^{2} \zeta_{\varepsilon}(x)\right)\right) d x \\
& -\int_{\Omega} \frac{1}{\varepsilon} \varphi_{\varepsilon}(x) \cdot \nabla\left(\operatorname{div}\left(\Delta^{-1}\left(\varepsilon^{2} \Delta \zeta_{\varepsilon}-\varphi_{\varepsilon}\right)\right)\right)(x) d x .
\end{aligned}
$$

The last integral can be estimated by

$$
\begin{aligned}
& \left|\int_{\Omega} \frac{1}{\varepsilon} \varphi_{\varepsilon}(x) \cdot \nabla\left(\operatorname{div}\left(\Delta^{-1}\left(\varepsilon^{2} \Delta \zeta_{\varepsilon}-\varphi_{\varepsilon}\right)\right)\right)(x) d x\right| \\
& \leq\left(\int_{\Omega} \frac{1}{\varepsilon}\left|\varphi_{\varepsilon}(x)\right|^{2}\right)^{1 / 2}\left(\frac{1}{\varepsilon} \int_{\mathbb{R}^{2}}\left|\nabla\left(\operatorname{div}\left(\Delta^{-1}\left(\varepsilon^{2} \Delta \zeta_{\varepsilon}-\varphi_{\varepsilon}\right)\right)\right)(x)\right|^{2} d x\right)^{1 / 2} \\
& \leq\left(\int_{\Omega} \frac{1}{\varepsilon}\left|\varphi_{\varepsilon}(x)\right|^{2}\right)^{1 / 2}\left(\frac{2}{\varepsilon} \int_{\mathbb{R}^{2}}\left|\nabla^{2}\left(\Delta^{-1}\left(\varepsilon^{2} \Delta \zeta_{\varepsilon}-\varphi_{\varepsilon}\right)\right)(x)\right|^{2} d x\right)^{1 / 2} \\
& =\left(\int_{\Omega} \frac{1}{\varepsilon}\left|\varphi_{\varepsilon}(x)\right|^{2}\right)^{1 / 2}\left(\int_{\Omega} \frac{2}{\varepsilon}\left|\varepsilon^{2} \Delta \zeta_{\varepsilon}(x)-\varphi_{\varepsilon}(x)\right|^{2} d x\right)^{1 / 2} .
\end{aligned}
$$

Then, since

$$
\begin{aligned}
\int_{\Omega} \frac{1}{\varepsilon}\left|\varphi_{\varepsilon}(x)\right|^{2} & \leq C \int_{\Omega} \frac{1}{\varepsilon}\left|\varphi_{\varepsilon}(x)\right| \\
& \leq \bar{C} \int_{\Omega} \frac{1}{\varepsilon}\left|\int_{B_{R}(0)} l(z, x)(\bar{\varphi}(x+\varepsilon z)-\varphi(x)) d z\right| d x \\
& \leq \bar{C} \int_{B_{R}(0)} \frac{1}{\varepsilon}|l(z, x)|\left(\int_{\Omega}|\bar{\varphi}(x+\varepsilon z)-\varphi(x)| d x\right) d z \\
& \leq \bar{C}\|D \bar{\varphi}\|\left(\mathbb{R}^{2}\right) \int_{B_{R}(0)}|l(z, x)| \cdot|z| d z=O(1)
\end{aligned}
$$

using (3.9), from (3.10) we infer

$$
\int_{\mathbb{R}^{2}} \frac{1}{\varepsilon}\left|\nabla\left(\operatorname{div}\left(\Delta^{-1} \varphi_{\varepsilon}\right)\right)(x)\right|^{2} d x=o_{\varepsilon}(1)+\int_{\Omega} \frac{1}{\varepsilon} \varphi_{\varepsilon}(x) \cdot \nabla\left(\operatorname{div}\left(\varepsilon^{2} \zeta_{\varepsilon}(x)\right)\right) d x .
$$

Next we remind that $\xi_{\varepsilon}$ is defined by (3.3). By (3.7), we have,

$$
\left|\nabla\left(\operatorname{div}\left(\varepsilon^{2} \zeta_{\varepsilon}(x)\right)\right)-\xi_{\varepsilon}(x)\right| \leq \bar{C} \varepsilon^{2 / 3} \quad \forall x \in \Omega .
$$


Then as before,

$$
\begin{aligned}
& \left|\int_{\Omega} \frac{1}{\varepsilon} \varphi_{\varepsilon}(x) \cdot\left(\nabla\left(\operatorname{div}\left(\varepsilon^{2} \zeta_{\varepsilon}(x)\right)\right)-\xi_{\varepsilon}(x)\right) d x\right| \\
& \leq\left(\int_{\Omega} \frac{1}{\varepsilon}\left|\varphi_{\varepsilon}(x)\right|^{2}\right)^{1 / 2}\left(\int_{\Omega} \frac{1}{\varepsilon}\left|\nabla\left(\operatorname{div}\left(\varepsilon^{2} \zeta_{\varepsilon}(x)\right)\right)-\xi_{\varepsilon}(x)\right|^{2} d x\right)^{1 / 2} \\
& \leq C \varepsilon^{1 / 6} .
\end{aligned}
$$

Therefore, from (3.12) we infer (3.4).

Lemma 3.2. Let $\varphi \in B V(\Omega, \mathbb{R}) \cap L^{\infty}$ and $l \in \mathcal{U}$ (see Definition 2.4). For every $\varepsilon>0$ and every $x \in \mathbb{R}^{2}$ consider the function $\varphi_{\varepsilon} \in C^{1}\left(\mathbb{R}^{2}, \mathbb{R}^{2}\right)$ as in (3.2). Then,

$$
\begin{aligned}
& \lim _{\varepsilon \rightarrow 0} \int_{\mathbb{R}^{2}} \frac{1}{\varepsilon}\left|\nabla\left(\operatorname{div}\left(\Delta^{-1} \varphi_{\varepsilon}\right)\right)(x)\right|^{2} d x \\
& =\int_{J_{\varphi}}\left\{\int_{-\infty}^{+\infty}\left|\varphi^{+}(x)-\varphi^{-}(x)\right|^{2} \cdot\left|\boldsymbol{v}(x) \cdot \int_{t}^{+\infty} q(s, x) d s\right|^{2} d t\right\} d \mathcal{H}^{1}(x),
\end{aligned}
$$

where

$$
q(t, x)=\int_{H_{\boldsymbol{v}(x)}^{0}} l(t \boldsymbol{v}(x)+y, x) d \mathcal{H}^{1}(y),
$$

and $\boldsymbol{v}(x)$ is the jump vector of $\varphi$.

Proof. By Lemma 3.1 we have

$$
\int_{\mathbb{R}^{2}} \frac{1}{\varepsilon}\left|\nabla\left(\operatorname{div}\left(\Delta^{-1} \varphi_{\varepsilon}\right)\right)(x)\right|^{2} d x=o_{\varepsilon}(1)+\int_{\Omega} \frac{1}{\varepsilon} \varphi_{\varepsilon}(x) \cdot \xi_{\varepsilon}(x) d x .
$$

From this point the strategy of the proof is similar to that in [16] and [17] (see also Proposition 2.2). The only difference is that here $\xi_{\varepsilon}$ is defined by a convolution of $\bar{\varphi}$ with a kernel whose support is not compact. However, it turns out that this difference is not crucial and we can use almost the same approach.

Step 1. We prove a useful expression,

$$
\begin{aligned}
& \int_{\Omega} \frac{1}{\varepsilon} \varphi_{\varepsilon}(x) \cdot \xi_{\varepsilon}(x) d x \\
& =\int_{0}^{1}\left\{\int_{\mathbb{R}^{2}}\left(\frac{1}{t^{2} \varepsilon^{2}} \int_{\Omega \cap B_{R t \varepsilon}(y)}\left\{\xi_{\varepsilon}(x) \cdot l\left(\frac{y-x}{t \varepsilon}, x\right)\right\} \frac{y-x}{t \varepsilon} d x\right) \cdot d[D \bar{\varphi}](y)\right\} d t,
\end{aligned}
$$


where $\xi_{\varepsilon}$ is as in (3.3) and $R>0$ is such that $l(z, x)=0$ whenever $|z| \geq R$. As before, we shall denote by $\nabla_{1} l$ and $\nabla_{2} l$ the gradient of $l$ with respect to the first and second variables respectively. Denote $\left(\varphi_{t \varepsilon, 1}(x), \varphi_{t \varepsilon, 2}(x)\right):=\varphi_{t \varepsilon}(x)$ and $\left(l_{1}(z, x), l_{2}(z, x)\right):=l(z, x)$. Then for every $t \in(0,1]$, every $j \in\{1,2\}$ and every $x \in \mathbb{R}^{2}$ we have

$$
\begin{aligned}
\frac{d\left(\varphi_{t \varepsilon, j}(x)\right)}{d t} & =\frac{d}{d t}\left(\frac{1}{t^{2} \varepsilon^{2}} \int_{\mathbb{R}^{2}} l_{j}\left(\frac{y-x}{t \varepsilon}, x\right) \bar{\varphi}(y) d y\right) \\
& =-\frac{1}{t^{3} \varepsilon^{2}} \int_{\mathbb{R}^{2}}\left\{\nabla_{1} l_{j}\left(\frac{y-x}{t \varepsilon}, x\right) \cdot \frac{y-x}{t \varepsilon}+2 l_{j}\left(\frac{y-x}{t \varepsilon}, x\right)\right\} \bar{\varphi}(y) d y \\
& =-\frac{1}{t^{2} \varepsilon} \int_{\mathbb{R}^{2}} \operatorname{div}_{y}\left\{l_{j}\left(\frac{y-x}{t \varepsilon}, x\right) \frac{y-x}{t \varepsilon}\right\} \bar{\varphi}(y) d y \\
& =\frac{1}{t^{2} \varepsilon} \int_{\mathbb{R}^{2}} l_{j}\left(\frac{y-x}{t \varepsilon}, x\right) \frac{y-x}{t \varepsilon} \cdot d[D \bar{\varphi}](y) .
\end{aligned}
$$

Therefore, for any $\rho \in(0,1)$ we have,

$$
\begin{aligned}
& \int_{\Omega} \frac{1}{\varepsilon}\left(\varphi_{\varepsilon}(x)-\varphi_{\rho \varepsilon}(x)\right) \cdot \xi_{\varepsilon}(x) d x \\
& =\int_{\Omega} \frac{1}{\varepsilon} \xi_{\varepsilon}(x) \cdot\left(\int_{\rho}^{1} \frac{d\left(\varphi_{t \varepsilon}(x)\right)}{d t}\right) d x \\
& =\int_{\Omega}\left\{\int_{\rho}^{1} \xi_{\varepsilon}(x) \cdot\left(\frac{1}{t^{2} \varepsilon^{2}} \int_{\mathbb{R}^{2}} l\left(\frac{y-x}{t \varepsilon}, x\right)\left\{\frac{y-x}{t \varepsilon} \cdot d[D \bar{\varphi}](y)\right\}\right) d t\right\} d x \\
& =\int_{\rho}^{1}\left\{\int_{\Omega} \xi_{\varepsilon}(x) \cdot\left(\frac{1}{t^{2} \varepsilon^{2}} \int_{\mathbb{R}^{2}} l\left(\frac{y-x}{t \varepsilon}, x\right)\left\{\frac{y-x}{t \varepsilon} \cdot d[D \bar{\varphi}](y)\right\}\right) d x\right\} d t \\
& =\int_{\rho}^{1}\left\{\int_{\mathbb{R}^{2}}\left(\frac{1}{t^{2} \varepsilon^{2}} \int_{\Omega \cap B_{R t \varepsilon}(y)}\left\{\xi_{\varepsilon}(x) \cdot l\left(\frac{y-x}{t \varepsilon}, x\right)\right\} \frac{y-x}{t \varepsilon} d x\right) \cdot d[D \bar{\varphi}](y)\right\} d t .
\end{aligned}
$$

From our assumptions on $\varphi$, by (3.1), it follows that there exists a constant $C>0$, independent of $\rho$, such that $\left|\xi_{\rho}(x)\right| \leq C$ for every $\rho>0$ and every $x \in \Omega$. Therefore, letting $\rho$ tend to zero in (3.19), using the fact that $\lim _{\rho \rightarrow 0}\left\|\varphi_{\rho}(x)\right\|_{L^{1}(\Omega)}=0$ (see (3.11)), we get (3.17). 
Step 2. We prove the identity

$$
\begin{aligned}
& \int_{\mathbb{R}^{2}} \frac{1}{\varepsilon}\left|\nabla\left(\operatorname{div}\left(\Delta^{-1} \varphi_{\varepsilon}\right)\right)(x)\right|^{2} d x \\
& =o_{\varepsilon}(1)+\iint_{0}^{1}\left(\int_{J_{\varphi}}\left\{\int_{B_{R}(0)}\left\{l(z, x) \cdot \xi_{\varepsilon}(x-\varepsilon t z)\right\} z d z\right\} \cdot d[D \varphi](x)\right) d t \\
& +\int_{0}^{1}\left(\int_{G_{\varphi}}\left\{\int_{B_{R}(0)}\left\{l(z, x) \cdot \xi_{\varepsilon}(x-\varepsilon t z)\right\} z d z\right\} \cdot d[D \varphi](x)\right) d t
\end{aligned}
$$

where $G_{\varphi}$ is the set of approximate continuity of $\varphi$. By (3.16) and (3.17) we deduce

$$
\begin{aligned}
& \int_{\mathbb{R}^{2}} \frac{1}{\varepsilon}\left|\nabla\left(\operatorname{div}\left(\Delta^{-1} \varphi_{\varepsilon}\right)\right)(x)\right|^{2} d x \\
& =o_{\varepsilon}(1)+\int_{0}^{1}\left\{\int_{\mathbb{R}^{2}}\left(\frac{1}{t^{2} \varepsilon^{2}} \int_{\Omega \cap B_{R t \varepsilon}(y)}\left\{\xi_{\varepsilon}(x) \cdot l\left(\frac{y-x}{t \varepsilon}, x\right)\right\} \frac{y-x}{t \varepsilon} d x\right) \cdot d[D \bar{\varphi}](y)\right\} d t \\
& =o_{\varepsilon}(1)+\int_{0}^{1}\left\{\int_{\mathbb{R}^{2}}\left(\frac{1}{t^{2} \varepsilon^{2}} \int_{K \cap B_{R t \varepsilon}(y)}\left\{\xi_{\varepsilon}(x) \cdot l\left(\frac{y-x}{t \varepsilon}, x\right)\right\} \frac{y-x}{t \varepsilon} d x\right) \cdot d[D \bar{\varphi}](y)\right\} d t,
\end{aligned}
$$

where $K \Subset \Omega$ is a compact set (see Definition 2.4). But, for every $\varepsilon<\frac{1}{R} \operatorname{dist}(K, \partial \Omega)$ we have

$$
\begin{aligned}
& \int_{\mathbb{R}^{2}}\left(\frac{1}{t^{2} \varepsilon^{2}} \int_{K \cap B_{R t \varepsilon}(y)}\left\{\xi_{\varepsilon}(x) \cdot l\left(\frac{y-x}{t \varepsilon}, x\right)\right\} \frac{y-x}{t \varepsilon} d x\right) \cdot d[D \bar{\varphi}](y) \\
& =\int_{\Omega}\left(\frac{1}{t^{2} \varepsilon^{2}} \int_{K \cap B_{R t \varepsilon}(y)}\left\{\xi_{\varepsilon}(x) \cdot l\left(\frac{y-x}{t \varepsilon}, x\right)\right\} \frac{y-x}{t \varepsilon} d x\right) \cdot d[D \bar{\varphi}](y) \\
& =\int_{\Omega}\left(\frac{1}{t^{2} \varepsilon^{2}} \int_{\mathbb{R}^{2}}\left\{\xi_{\varepsilon}(x) \cdot l\left(\frac{y-x}{t \varepsilon}, x\right)\right\} \frac{y-x}{t \varepsilon} d x\right) \cdot d[D \bar{\varphi}](y) .
\end{aligned}
$$


Therefore, by (3.21), we obtain

$$
\begin{aligned}
& \int_{\mathbb{R}^{2}} \frac{1}{\varepsilon}\left|\nabla\left(\operatorname{div}\left(\Delta^{-1} \varphi_{\varepsilon}\right)\right)(x)\right|^{2} d x \\
& =o_{\varepsilon}(1)+\int_{0}^{1} \iint_{\Omega}\left(\frac{1}{t^{2} \varepsilon^{2}} \iint_{\mathbb{R}^{2}}\left\{\xi_{\varepsilon}(x) \cdot l\left(\frac{y-x}{t \varepsilon}, x\right)\right\} \frac{y-x}{t \varepsilon} d x\right) \cdot d[D \bar{\varphi}](y) d t \\
& =o_{\varepsilon}(1)+\int_{0}^{1}\left(\int_{\Omega}\left\{\int_{B_{R}(0)}\left\{l(z, y-\varepsilon t z) \cdot \xi_{\varepsilon}(y-\varepsilon t z)\right\} z d z\right\} \cdot d[D \varphi](y)\right) d t \\
& \left.=o_{\varepsilon}(1)+\int_{0}^{1}\left(\int_{\Omega} \int_{B_{R}(0)}\left\{l(z, x) \cdot \xi_{\varepsilon}(x-\varepsilon t z)\right\} z d z\right\} \cdot d[D \varphi](x)\right) d t,
\end{aligned}
$$

where in the last equality we used the estimate $|l(z, x-\varepsilon t z)-l(z, x)| \leq C \varepsilon t|z|$. Therefore we obtain (3.20).

Step 3. We will prove that the second integral in the r.h.s of (3.20) vanishes as $\varepsilon \rightarrow 0$. For every $x$ in $G_{\varphi}$ we have,

$$
\lim _{\rho \rightarrow 0^{+}} \frac{1}{\rho^{2}} \int_{B_{\rho}(x)}|\bar{\varphi}(y)-\tilde{\bar{\varphi}}(x)| d y=0 .
$$

Taking $\rho=L \varepsilon$, for every $L>0$, gives

$$
\lim _{\varepsilon \rightarrow 0^{+}} \int_{B_{L}(0)}|\bar{\varphi}(x+\varepsilon z)-\tilde{\bar{\varphi}}(x)| d z=0, \text { for } x \text { in } G_{\varphi} .
$$

Using (3.1), since $\int_{\mathbb{R}^{2}} \nabla_{z}\left(\operatorname{div}_{z} r(z, x-\varepsilon t y)\right) d z=0$, for every $x$ in $G_{\varphi}, y \in B_{R}(0)$, $t \in[0,1]$ and $L>0$ we have,

$$
\begin{aligned}
& \left|\xi_{\varepsilon}(x-\varepsilon t y)\right|=\left|\int_{\mathbb{R}^{2}} \nabla_{z}\left(\operatorname{div}_{z} r(z, x-\varepsilon t y)\right) \bar{\varphi}(x+\varepsilon z-\varepsilon t y) d z\right| \\
& =\left|\int_{\mathbb{R}^{2}} \nabla_{z}\left(\operatorname{div}_{z} r(z, x-\varepsilon t y)\right)(\bar{\varphi}(x+\varepsilon z-\varepsilon t y)-\tilde{\bar{\varphi}}(x)) d z\right| \\
& \leq \int_{B_{L}(0)}\left|\nabla_{z}\left(\operatorname{div}_{z} r(z, x-\varepsilon t y)\right)\right| \cdot|\bar{\varphi}(x+\varepsilon z-\varepsilon t y)-\tilde{\bar{\varphi}}(x)| d z \\
& \quad+\int_{\mathbb{R}^{2} \backslash B_{L}(0)}\left|\nabla_{z}\left(\operatorname{div}_{z} r(z, x-\varepsilon t y)\right)\right| \cdot|\bar{\varphi}(x+\varepsilon z-\varepsilon t y)-\tilde{\bar{\varphi}}(x)| d z \\
& \leq A_{L} \int_{B_{L}(0)}|\bar{\varphi}(x+\varepsilon z-\varepsilon t y)-\tilde{\bar{\varphi}}(x)| d z+B \int_{\mathbb{R}^{2} \backslash B_{L}(0)} \frac{1}{|z|^{3}+1} d z \\
& \leq A_{L} \int_{B_{(L+R)}(0)}|\bar{\varphi}(x+\varepsilon z)-\tilde{\bar{\varphi}}(x)| d z+B \int_{\mathbb{R}^{2} \backslash B_{L}(0)} \frac{1}{|z|^{3}+1} d z,
\end{aligned}
$$


where $B>0$ is a constant and $A_{L}>0$ depends only on $L$. Given $\delta>0$ we can take $L>0$ such that

$$
B \int_{\mathbb{R}^{2} \backslash B_{L}(0)} \frac{1}{|z|^{3}+1} d z<\delta,
$$

Then, using (3.24) and (3.23), we infer $\varlimsup_{\varepsilon \rightarrow 0^{+}}\left|\xi_{\varepsilon}(x-\varepsilon t y)\right|<\delta$ and since $\delta$ was arbitrary,

$$
\lim _{\varepsilon \rightarrow 0^{+}} \xi_{\varepsilon}(x-\varepsilon t y)=0 \quad \forall x \in G_{\varphi}, y \in B_{R}(0), t \in[0,1] .
$$

Using (3.1), we also have $\left|\xi_{\varepsilon}(x-\varepsilon t y)\right| \leq C$, and therefore, plugging (3.25) into (3.20), we obtain

$$
\begin{aligned}
& \int_{\mathbb{R}^{2}} \frac{1}{\varepsilon}\left|\nabla\left(\operatorname{div}\left(\Delta^{-1} \varphi_{\varepsilon}\right)\right)(x)\right|^{2} d x \\
& =o_{\varepsilon}(1)+\int_{0}^{1}\left(\int_{J_{\varphi}}\left\{\int_{B_{R}(0)}\left\{l(z, x) \cdot \xi_{\varepsilon}(x-\varepsilon t z)\right\} z d z\right\} \cdot d[D \varphi](x)\right) d t .
\end{aligned}
$$

Step 4. Consider $\bar{l}(z, x):=\nabla_{z}\left(\operatorname{div}_{z} r(z, x)\right)$. For every $\varepsilon, t \in(0,1), x \in J_{\varphi}$ and $z \in B_{R}(0)$, we have

$$
\begin{aligned}
\xi_{\varepsilon}(x-\varepsilon t z)= & \int_{\mathbb{R}^{2}} \bar{l}(y, x-\varepsilon t z) \bar{\varphi}(x+\varepsilon(y-t z)) d y \\
= & \int_{\mathbb{R}^{2}} \bar{l}(y+t z, x-\varepsilon t z) \bar{\varphi}(x+\varepsilon y) d y \\
= & \int_{H_{+}(0, \boldsymbol{v}(x))} \bar{l}(y+t z, x-\varepsilon t z) \bar{\varphi}(x+\varepsilon y) d y \\
& +\int_{H_{-}(0, \boldsymbol{v}(x))} \bar{l}(y+t z, x-\varepsilon t z) \bar{\varphi}(x+\varepsilon y) d y \\
= & \int_{H_{+}} \bar{l}(y, \boldsymbol{v}(x)) \\
& +\int_{H_{-}(0, \boldsymbol{v}(x))} \bar{l}(y+t z, x-\varepsilon t z) \varphi^{-}(x) d y \\
& +\int_{H_{+}(0, \boldsymbol{v}(x))} \bar{l}(y+t z, x-\varepsilon t z)\left(\bar{\varphi}(x+\varepsilon y)-\varphi^{+}(x)\right) d y \\
& +\int_{H_{-}(0, \boldsymbol{v}(x))} \bar{l}(y+t z, x-\varepsilon t z)\left(\bar{\varphi}(x+\varepsilon y)-\varphi^{-}(x)\right) d y .
\end{aligned}
$$


By the definition of $J_{\varphi}$, for every $L>0$ we obtain,

$$
\begin{array}{ll}
\lim _{\varepsilon \rightarrow 0^{+}} \int_{B_{L}^{+}(0, \boldsymbol{v}(x))}\left|\varphi(x+\varepsilon z)-\varphi^{+}(x)\right| d z=0, & \\
\lim _{\varepsilon \rightarrow 0^{+}} \int_{B_{L}^{-}(0, \boldsymbol{v}(x))}\left|\varphi(x+\varepsilon z)-\varphi^{-}(x)\right| d z=0 . & \text { for } x \in J_{\varphi} .
\end{array}
$$

Then, by (3.1), for every $L>R$ we have,

$$
\begin{aligned}
& \left|\int_{H_{+}(0, \boldsymbol{v}(x))} \bar{l}(y+t z, x-\varepsilon t z)\left(\bar{\varphi}(x+\varepsilon y)-\varphi^{+}(x)\right) d y\right| \\
& \leq \int_{B_{L}^{+}(0, \boldsymbol{v}(x))}|\bar{l}(y+t z, x-\varepsilon t z)| \cdot\left|\bar{\varphi}(x+\varepsilon y)-\varphi^{+}(x)\right| d y \\
& \quad+\int_{H_{+}(0, \boldsymbol{v}(x)) \backslash B_{L}^{+}(0, \boldsymbol{v}(x))}|\bar{l}(y+t z, x-\varepsilon t z)| \cdot\left|\bar{\varphi}(x+\varepsilon y)-\varphi^{+}(x)\right| d y \\
& \leq A_{L} \int_{B_{L}^{+}(0, \boldsymbol{v}(x))}\left|\bar{\varphi}(x+\varepsilon y)-\varphi^{+}(x)\right| d y+B \int_{\mathbb{R}^{2} \backslash B_{L}(0)} \frac{1}{(|y|-R)^{3}+1} d y,
\end{aligned}
$$

where $B>0$ is a constant and $A_{L}>0$ depends only on $L$. Given $\delta>0$ we can take $L>0$ such that

$$
B \int_{\mathbb{R}^{2} \backslash B_{L}(0)} \frac{1}{(|y|-R)^{3}+1} d y<\delta,
$$

Then, using (3.29) and (3.28), we infer

$$
\varlimsup_{\varepsilon \rightarrow 0^{+}}\left|\int_{H_{+}(0, \boldsymbol{v}(x))} \bar{l}(y+t z, x-\varepsilon t z)\left(\bar{\varphi}(x+\varepsilon y)-\varphi^{+}(x)\right) d y\right|<\delta,
$$

and since $\delta$ was arbitrary,

$$
\begin{gathered}
\lim _{\varepsilon \rightarrow 0^{+}} \int_{H_{+}(0, \boldsymbol{v}(x))} \bar{l}(y+t z, x-\varepsilon t z)\left(\bar{\varphi}(x+\varepsilon y)-\varphi^{+}(x)\right) d y=0 \\
\forall x \in J_{\varphi}, z \in B_{R}(0), t \in[0,1] .
\end{gathered}
$$

By the same method,

$$
\begin{gathered}
\lim _{\varepsilon \rightarrow 0^{+}} \int_{H_{-}(0, \boldsymbol{v}(x))} \bar{l}(y+t z, x-\varepsilon t z)\left(\bar{\varphi}(x+\varepsilon y)-\varphi^{-}(x)\right) d y=0 \\
\forall x \in J_{\varphi}, z \in B_{R}(0), t \in[0,1] .
\end{gathered}
$$


Therefore, by (3.27) for every $\varepsilon, t \in(0,1), x \in J_{\varphi}$ and $z \in B_{R}(0)$, we have

$$
\begin{aligned}
\xi_{\varepsilon}(x-\varepsilon t z)= & o_{\varepsilon}(1)+\varphi^{+}(x) \\
& \times \int_{H_{+}(0, \boldsymbol{v}(x))} \bar{l}(y+t z, x-\varepsilon t z) d y+\varphi^{-}(x) \int_{H_{-}(0, \boldsymbol{v}(x))} \bar{l}(y+t z, x-\varepsilon t z) d y \\
= & o_{\varepsilon}(1)+\left(\varphi^{+}(x)-\varphi^{-}(x)\right) \int_{H_{+}(0, \boldsymbol{v}(x))} \bar{l}(y+t z, x-\varepsilon t z) d y,
\end{aligned}
$$

where we used the equality $\int_{\mathbb{R}^{2}} \bar{l}(y+t z, x-\varepsilon t z) d y=0$. Using (3.1), gives

$$
\lim _{\varepsilon \rightarrow 0^{+}} \int_{H_{+}(0, \boldsymbol{v}(x))} \bar{l}(y+t z, x-\varepsilon t z) d y=\int_{H_{+}(0, \boldsymbol{v}(x))} \bar{l}(y+t z, x) d y .
$$

Therefore, by (3.32), for every $x \in J_{\varphi}$, every $t \in(0,1)$ and every $z \in B_{R}(0)$, we obtain,

$$
\lim _{\varepsilon \rightarrow 0^{+}} \xi_{\varepsilon}(x-\varepsilon t z)=\left(\varphi^{+}(x)-\varphi^{-}(x)\right) \int_{H_{+}(0, \boldsymbol{v}(x))} \bar{l}(y+t z, x) d y .
$$

Note that

$$
\begin{aligned}
\int_{H_{+}(0, \boldsymbol{v}(x))} \bar{l}(y+t z, x) d y & =\int_{H_{+}(t z, \boldsymbol{v}(x))} \bar{l}(y, x) d y \\
& =\int_{t \boldsymbol{v}(x) \cdot z}^{+\infty}\left(\int_{H_{\boldsymbol{v}(x)}^{0}} \bar{l}(t \boldsymbol{v}(x)+y, x) d \mathcal{H}^{1}(y)\right) d t \\
& =\int_{t \boldsymbol{v}(x) \cdot z}^{+\infty} \bar{q}(\tau, x) d \tau,
\end{aligned}
$$

where

$$
\bar{q}(t, x)=\int_{H_{v(x)}^{0}} \bar{l}(t \boldsymbol{v}(x)+y, x) d \mathcal{H}^{1}(y) .
$$

Combining (3.33) and (3.34), for every $x \in J_{\varphi}$, every $t \in(0,1)$ and every $z \in$ $B_{R}(0)$ we obtain,

$$
\lim _{\varepsilon \rightarrow 0+} \xi_{\varepsilon}(x-\varepsilon t z)=\left(\varphi^{+}(x)-\varphi^{-}(x)\right) \int_{t v(x) \cdot z}^{+\infty} \bar{q}(\tau, x) d \tau
$$


Using (3.36) in (3.26), we obtain,

$$
\begin{aligned}
& \int_{\mathbb{R}^{2}} \frac{1}{\varepsilon}\left|\nabla\left(\operatorname{div}\left(\Delta^{-1} \varphi_{\varepsilon}\right)\right)(x)\right|^{2} d x=o_{\varepsilon}(1) \\
& +\int_{0}^{1}\left(\int_{J_{\varphi}}\left(\varphi^{+}(x)-\varphi^{-}(x)\right)\left\{\int_{B_{R}(0)}\left(l(z, x) \cdot \int_{t v(x) z}^{+\infty} \bar{q}(\tau, x) d \tau\right) z d z\right\} \cdot d[D \varphi](x)\right) d t \\
& =o_{\varepsilon}(1) \\
& +\int_{J_{\varphi}}\left|\varphi^{+}(x)-\varphi^{-}(x)\right|^{2}\left\{\int_{B_{R}(0)}\left(l(z, x) \cdot \int_{0}^{1} \int_{t v(x) \cdot z}^{+\infty} \bar{q}(\tau, x) d \tau d t\right)(v(x) \cdot z) d z\right\} d \mathcal{H}^{1}(x) .
\end{aligned}
$$

Step 5. We prove that

$$
\bar{q}(t, x)=(q(t, x) \cdot v(x)) v(x) .
$$

Consider $\left(r_{1}(z, x), r_{2}(z, x)\right):=r(z, x)$. Then, by (3.1), for every $k=1,2$, we obtain,

$$
\begin{aligned}
& \int_{H_{\boldsymbol{v}(x)}^{0}} \nabla_{z}^{2} r_{k}(t \boldsymbol{v}(x)+y, x) d \mathcal{H}^{1}(y)=\int_{H_{\boldsymbol{v}(x)}^{0}} \boldsymbol{v}(x) \otimes \boldsymbol{v}(x) \frac{\partial^{2} r_{k}}{\partial(\boldsymbol{v}(x))^{2}}(t \boldsymbol{v}(x)+y, x) d \mathcal{H}^{1}(y) \\
& \quad+\int_{H_{\boldsymbol{v}(x)}^{0}}\left(\boldsymbol{v}(x) \otimes \boldsymbol{v}^{\perp}(x)+\boldsymbol{v}^{\perp}(x) \otimes \boldsymbol{v}(x)\right) \frac{\partial^{2} r_{k}}{\partial\left(\boldsymbol{v}^{\perp}(x)\right) \partial(\boldsymbol{v}(x))}(t \boldsymbol{v}(x)+y, x) d \mathcal{H}^{1}(y) \\
& \quad+\int_{H_{\boldsymbol{v}(x)}^{0}} \boldsymbol{v}^{\perp}(x) \otimes \boldsymbol{v}^{\perp}(x) \frac{\partial^{2} r_{k}}{\partial\left(\boldsymbol{v}^{\perp}(x)\right)^{2}}(t \boldsymbol{v}(x)+y, x) d \mathcal{H}^{1}(y) \\
& =\int_{H_{\boldsymbol{v}(x)}^{0} \boldsymbol{v}(x) \otimes \boldsymbol{v}(x) \frac{\partial^{2} r_{k}}{\partial(\boldsymbol{v}(x))^{2}}(t \boldsymbol{v}(x)+y, x) d \mathcal{H}^{1}(y),}
\end{aligned}
$$

where $\boldsymbol{v}^{\perp}(x)$ is the vector orthogonal to $\boldsymbol{v}(x)$ in $\mathbb{R}^{2}$ and all derivatives are taken in the first argument- $z$ of $r(z, x)$. In particular

$$
\begin{aligned}
q(t, x) & =\int_{H_{\boldsymbol{v}(x)}^{0}} l(t \boldsymbol{v}(x)+y, x) d \mathcal{H}^{1}(y)=\int_{H_{\boldsymbol{v}(x)}^{0}} \Delta r(t \boldsymbol{v}(x)+y, x) d \mathcal{H}^{1}(y) \\
& =\int_{H_{\boldsymbol{v}(x)}^{0}} \frac{\partial^{2} r}{\partial(\boldsymbol{v}(x))^{2}}(t \boldsymbol{v}(x)+y, x) d \mathcal{H}^{1}(y),
\end{aligned}
$$


and

$$
\begin{aligned}
\bar{q}(t, x) & =\int_{H_{\boldsymbol{v}(x)}^{0}} \bar{l}(t \boldsymbol{v}(x)+y, x) d \mathcal{H}^{1}(y)=\int_{H_{\boldsymbol{v}(x)}^{0}} \nabla_{z}\left(\operatorname{div}_{z} r\right)(t \boldsymbol{v}(x)+y, x) d \mathcal{H}^{1}(y) \\
& =\left(\boldsymbol{v}(x) \cdot \int_{H_{\boldsymbol{v}(x)}^{0}} \frac{\partial^{2} r}{\partial(\boldsymbol{v}(x))^{2}}(t \boldsymbol{v}(x)+y, x) d \mathcal{H}^{1}(y)\right) \boldsymbol{v}(x)
\end{aligned}
$$

So, we obtain (3.38).

Step 6. Completing the proof. Plugging (3.38) into (3.37) gives

$$
\begin{aligned}
& \int_{\mathbb{R}^{2}} \frac{1}{\varepsilon}\left|\nabla\left(\operatorname{div}\left(\Delta^{-1} \varphi_{\varepsilon}\right)\right)(x)\right|^{2} d x=o_{\varepsilon}(1)+\int_{J_{\varphi}}\left|\varphi^{+}(x)-\varphi^{-}(x)\right|^{2} \\
& \times\left\{\int_{B_{R}(0)}\left((l(z, x) \cdot \boldsymbol{v}(x)) \int_{0}^{1} \int_{t \boldsymbol{v}(x) \cdot z}^{+\infty}(q(\tau, x) \cdot \boldsymbol{v}(x)) d \tau d t\right)(v(x) \cdot z) d z\right\} d \mathcal{H}^{1}(x) .
\end{aligned}
$$

Next we have

$$
\begin{aligned}
& \int_{B_{R}(0)}\left((l(z, x) \cdot \boldsymbol{v}(x)) \int_{0}^{1} \int_{t \boldsymbol{v}(x) \cdot z}^{+\infty}(q(\tau, x) \cdot \boldsymbol{v}(x)) d \tau d t\right)(v(x) \cdot z) d z \\
= & \int_{\mathbb{R}^{2}}\left((l(z, x) \cdot \boldsymbol{v}(x)) \int_{0}^{1} \int_{t \boldsymbol{v}(x) \cdot z}^{+\infty}(q(\tau, x) \cdot \boldsymbol{v}(x)) d \tau d t\right)(v(x) \cdot z) d z \\
= & \int_{-\infty}^{+\infty} s\left(\int_{0}^{1+\infty}(q(\tau, x) \cdot \boldsymbol{v}(x)) d \tau d t\right)\left(\int_{t s}(l(s \boldsymbol{v}(x)+y, x) \cdot \boldsymbol{v}(x)) d \mathcal{H}^{1}(y)\right) d s \\
= & \int_{-\infty}^{+\infty} s(q(s, x) \cdot \boldsymbol{v}(x))\left(\int_{0}^{0} \int_{t s}^{1}(q)\right. \\
& \int_{-\infty}^{+\infty}(q(s, x) \cdot \boldsymbol{v}(x))\left(\int_{0}^{s} \int_{t}^{+\infty}(q(\tau, x) \cdot \boldsymbol{v}(x)) d \tau d t\right) d s .
\end{aligned}
$$


Using the fact that $\int_{\mathbb{R}} q(\tau, x) d \tau=0$ and integrating by path, we obtain,

$$
\begin{aligned}
& \int_{-\infty}^{+\infty}(q(s, x) \cdot \boldsymbol{v}(x))\left(\int_{0}^{s} \int_{t}^{+\infty}(q(\tau, x) \cdot \boldsymbol{v}(x)) d \tau d t\right) d s \\
& =\int_{-\infty}^{+\infty}\left(\int_{s}^{+\infty}(q(\tau, x) \cdot \boldsymbol{v}(x)) d \tau\right)^{2} d s .
\end{aligned}
$$

Therefore, returning to (3.41) we infer

$$
\begin{aligned}
& \int_{B_{R}(0)}\left((l(z, x) \cdot \boldsymbol{v}(x)) \int_{0}^{1} \int_{t \boldsymbol{v}(x) \cdot z}^{+\infty}(q(\tau, x) \cdot \boldsymbol{v}(x)) d \tau d t\right)(v(x) \cdot z) d z \\
= & \int_{-\infty}^{+\infty}\left(\int_{t}^{+\infty}(q(s, x) \cdot v(x)) d s\right)^{2} d t
\end{aligned}
$$

Plugging (3.43) in (3.40) gives the desired result (3.14).

\section{Proof of the main result}

As before, throughout this section we assume that $\Omega$ is a bounded domain in $\mathbb{R}^{2}$ with Lipschitz boundary. Next consider $u \in B V\left(\Omega, S^{1}\right)$, satisfying $\operatorname{div} u=0$ in $\Omega$ and $u \cdot \boldsymbol{n}=0$ on $\partial \Omega(\boldsymbol{n}$ is the unit normal to $\partial \Omega)$. Let $\varphi \in B V(\Omega, \mathbb{R}) \cap L^{\infty}(\Omega, \mathbb{R})$, satisfying $u=e^{i \varphi}$ a e. in $\Omega$. By [2, Proposition 3.21] we may extend $\varphi$ to a function $\bar{\varphi} \in B V\left(\mathbb{R}^{2}, \mathbb{R}\right) \cap L^{\infty}\left(\mathbb{R}^{2}, \mathbb{R}\right)$ satisfying $\bar{\varphi}=\varphi$ a.e. in $\Omega$, supp $\bar{\varphi}$ is compact and $\|D \bar{\varphi}\|(\partial \Omega)=0$ (from the proof of Proposition 3.21 in [2] it follows that if $\varphi$ is bounded then its extension is also bounded). We also denote by $\bar{u}:=e^{i \bar{\varphi}}$. Then $\bar{u} \in B V\left(\Omega^{\prime \prime}, \mathbb{R}^{2}\right) \cap L^{\infty}\left(\Omega^{\prime \prime}, \mathbb{R}^{2}\right)$ for some $\Omega^{\prime \prime} \ni \Omega$, satisfying $\bar{u}=u$ a.e. in $\Omega$ and, by Volpert's chain rule, $\|D \bar{u}\|(\partial \Omega)=0$. Consider $\eta \in \mathcal{V}$. For any $\varepsilon>0$ define a function $\psi_{\varepsilon}(x): \mathbb{R}^{2} \rightarrow \mathbb{R}$ by

$$
\psi_{\varepsilon}(x):=\frac{1}{\varepsilon^{2}} \int_{\mathbb{R}^{2}} \eta\left(\frac{y-x}{\varepsilon}, x\right) \bar{\varphi}(y) d y=\int_{\mathbb{R}^{2}} \eta(z, x) \bar{\varphi}(x+\varepsilon z) d z, \quad \forall x \in \mathbb{R}^{2} .
$$

Proposition 4.1. Let $u, \varphi, \bar{u}, \bar{\varphi}$ and $\eta$ be as above. Consider $\psi_{\varepsilon}(x)$ defined by (4.1). Then,

$$
\begin{aligned}
& \lim _{\varepsilon \rightarrow 0} \int_{\mathbb{R}^{2}}\left|\nabla \operatorname{div} \Delta^{-1}\left(\chi_{\Omega}(x) e^{i \psi_{\varepsilon}(x)}\right)\right|^{2} d x \\
& =\int_{J_{\varphi}}\left\{\int_{-\infty}^{+\infty}\left|\boldsymbol{v}(x) \cdot\left(e^{i \gamma(t, x)}-e^{i \varphi^{-}(x)}\right)\right|^{2} d t\right\} d \mathcal{H}^{1}(x),
\end{aligned}
$$

where

$$
\gamma(t, x)=\varphi^{-}(x) \int_{-\infty}^{t} p(s, x) d s+\varphi^{+}(x) \int_{t}^{+\infty} p(s, x) d s
$$


with

$$
p(t, x)=\int_{H_{\boldsymbol{v}(x)}^{0}} \eta(t \boldsymbol{v}(x)+y, x) d \mathcal{H}^{1}(y),
$$

and $\chi_{\Omega}$ is the characteristic function of $\Omega$.

Proof. We follow basically the strategy that was described in Subsection 1.1.

Since $\left(u^{+}-u^{-}\right) \cdot \boldsymbol{v}=0$, the right hand side in (4.2) does not depend on the orientation of $J_{\varphi}$, we may assume that $\boldsymbol{v}(x)$ is Borel measurable. Together with $\eta \in \mathcal{V}$ we consider a second kernel $\bar{\eta} \in \mathcal{V}$. Let

$$
\bar{p}(t, x)=\int_{H_{\boldsymbol{v}(x)}^{0}} \bar{\eta}(t \boldsymbol{v}(x)+y, x) d \mathcal{H}^{1}(y) .
$$

For any $\varepsilon>0$ define a function $u_{\varepsilon}(x): \mathbb{R}^{2} \rightarrow \mathbb{R}^{2}$ by

$$
u_{\varepsilon}(x):=\frac{1}{\varepsilon^{2}} \int_{\mathbb{R}^{2}} \bar{\eta}\left(\frac{y-x}{\varepsilon}, x\right) \bar{u}(y) d y=\int_{\mathbb{R}^{2}} \bar{\eta}(z, x) e^{i \bar{\varphi}(x+\varepsilon z)} d z, \quad \forall x \in \mathbb{R}^{2} .
$$

Define $Q: \mathbb{R} \times J_{\varphi} \rightarrow \mathbb{R}^{2}$ by

$$
Q(t, x):=e^{i \gamma(t, x)}-\left(\left\{\int_{-\infty}^{t} \bar{p}(s, x) d s\right\} e^{i \varphi^{-}(x)}+\left\{\int_{t}^{+\infty} \bar{p}(s, x) d s\right\} e^{i \varphi^{+}(x)}\right),
$$

where $\gamma(t, x)$ is defined by (4.3). Then define $q: \mathbb{R} \times \Omega \rightarrow \mathbb{R}^{2}$ by

$$
q(t, x)= \begin{cases}-\frac{1}{\left(\varphi^{+}(x)-\varphi^{-}(x)\right)} \frac{d Q(t, x)}{d t} & x \in J_{\varphi}, \\ 0 & x \in \Omega \backslash J_{\varphi} .\end{cases}
$$

Then $q(t, x)$ is Borel measurable, $q$ is bounded on $\mathbb{R} \times \Omega$, there exists $M>0$ such that supp $q \subset[-M, M] \times \Omega$ and $\int_{\mathbb{R}} q(t, x) d t=0 \forall x \in \Omega$. Moreover

$$
\left(\varphi^{+}(x)-\varphi^{-}(x)\right) \int_{t}^{+\infty} q(s, x) d s=Q(t, x) .
$$

Then by Lemma 2.6, there exists a sequence of functions $l_{n} \in \mathcal{U}$ (see Definition 2.4), such that the sequence of functions $\left\{q_{n}\right\}$ defined on $\mathbb{R} \times \Omega$ by

$$
q_{n}(t, x)=\int_{H_{v_{0}(x)}^{0}} l_{n}\left(t \boldsymbol{v}_{0}(x)+y, x\right) d \mathcal{H}^{1}(y),
$$

has the following properties:

there exists $C_{0}$ such that $\left\|q_{n}\right\|_{L^{\infty}} \leq C_{0}$,

there exists $M>0$ such that $q_{n}(t, x)=0$ for $|t|>M$, and every $x \in \Omega$,

$$
\lim _{n \rightarrow \infty} \int_{\Omega} \int_{\mathbb{R}}\left|q_{n}(t, x)-q(t, x)\right| d t d\|D \varphi\|(x)=0 \text {. }
$$


In particular,

$$
\lim _{n \rightarrow \infty} \int_{J_{\varphi}} \int_{\mathbb{R}}\left|\varphi^{+}(x)-\varphi^{-}(x)\right| \cdot\left|q_{n}(t, x)-q(t, x)\right| d t d \mathcal{H}^{1}(x)=0 .
$$

For every positive integer $n$ and for every $\varepsilon>0$ consider the function $\varphi_{n, \varepsilon} \in$ $C^{1}\left(\mathbb{R}^{2}, \mathbb{R}^{2}\right)$ given by

$$
\varphi_{n, \varepsilon}(x):=\frac{1}{\varepsilon^{2}} \int_{\mathbb{R}^{2}} l_{n}\left(\frac{y-x}{\varepsilon}, x\right) \bar{\varphi}(y) d y=\int_{\mathbb{R}^{2}} l_{n}(z, x) \bar{\varphi}(x+\varepsilon z) d z,
$$

Next, we will use the following inequality, valid $\forall f(x), g(x), \lambda(x) \in L^{2}\left(\mathbb{R}^{2}, \mathbb{R}^{2}\right)$,

$$
\begin{aligned}
& \left.\left|\int_{\mathbb{R}^{2}}\right| f(x)\right|^{2} d x-\int_{\mathbb{R}^{2}}|g(x)|^{2} d x \mid \\
& \quad \leq\left(\|f-g-\lambda\|_{L^{2}}+\|\lambda\|_{L^{2}}\right) \sqrt{2\left(\int_{\mathbb{R}^{2}}|f(x)|^{2} d x+\int_{\mathbb{R}^{2}}|g(x)|^{2} d x\right)} .
\end{aligned}
$$

Therefore, since $\varphi_{n, \varepsilon}(x)=0$ for $x \notin \Omega$ and since $\operatorname{div}\left(\chi_{\Omega} \bar{u}\right)=0$ as a distribution, we obtain,

$$
\begin{aligned}
& \left.\left|\int_{\mathbb{R}^{2}}\right| \nabla \operatorname{div} \Delta^{-1}\left(\chi_{\Omega}(x) e^{i \psi_{\varepsilon}(x)}\right)\right|^{2} d x-\int_{\mathbb{R}^{2}}\left|\nabla \operatorname{div} \Delta^{-1}\left(\varphi_{n, \varepsilon}(x)\right)\right|^{2} d x \mid \\
& \leq 2\left(\left\|\nabla \operatorname{div} \Delta^{-1}\left(\chi_{\Omega}\left(e^{i \psi_{\varepsilon}}-\varphi_{n, \varepsilon}-u_{\varepsilon}\right)\right)\right\|_{L^{2}}+\left\|\nabla \operatorname{div} \Delta^{-1}\left(\chi_{\Omega} u_{\varepsilon}\right)\right\|_{L^{2}}\right) \\
& \times \sqrt{\int_{\mathbb{R}^{2}}\left|\nabla \operatorname{div} \Delta^{-1}\left(\chi_{\Omega} e^{i \psi_{\varepsilon}}\right)\right|^{2} d x+\int_{\mathbb{R}^{2}}\left|\nabla \operatorname{div} \Delta^{-1}\left(\varphi_{n, \varepsilon}\right)\right|^{2} d x} \\
& =2\left(\left\|\nabla \operatorname{div} \Delta^{-1}\left(\chi_{\Omega}\left(e^{i \psi_{\varepsilon}}-\varphi_{n, \varepsilon}-u_{\varepsilon}\right)\right)\right\|_{L^{2}}+\left\|\nabla \operatorname{div} \Delta^{-1}\left(\chi_{\Omega}\left(u_{\varepsilon}-\bar{u}\right)\right)\right\|_{L^{2}}\right) \\
& \quad \times \sqrt{\int_{\mathbb{R}^{2}}\left|\nabla \operatorname{div} \Delta^{-1}\left(\chi_{\Omega}\left(e^{i \psi_{\varepsilon}}-e^{i \bar{\varphi}}\right)\right)\right|^{2} d x+\int_{\mathbb{R}^{2}}\left|\nabla \operatorname{div} \Delta^{-1}\left(\varphi_{n, \varepsilon}\right)\right|^{2} d x} .
\end{aligned}
$$

But since for every $f \in L^{\infty}\left(\mathbb{R}^{2}, \mathbb{R}^{2}\right)$ with compact support we have

$$
\int_{\mathbb{R}^{2}}\left|\nabla \operatorname{div} \Delta^{-1} f\right|^{2} d x \leq 2 \int_{\mathbb{R}^{2}}\left|\nabla^{2} \Delta^{-1} f\right|^{2} d x=2 \int_{\mathbb{R}^{2}}|f|^{2} d x,
$$


by (4.16), we obtain

$$
\begin{aligned}
\left|\frac{1}{\varepsilon} \int_{\mathbb{R}^{2}}\right| \nabla & \left.\operatorname{div} \Delta^{-1}\left(\chi_{\Omega}(x) e^{i \psi_{\varepsilon}(x)}\right)\right|^{2} d x-\frac{1}{\varepsilon} \int_{\mathbb{R}^{2}}\left|\nabla \operatorname{div} \Delta^{-1}\left(\varphi_{n, \varepsilon}(x)\right)\right|^{2} d x \mid \\
\leq & 4\left(\sqrt{\frac{1}{\varepsilon} \int_{\Omega}\left|e^{i \psi_{\varepsilon}}-\varphi_{n, \varepsilon}-u_{\varepsilon}\right|^{2} d x}+\sqrt{\frac{1}{\varepsilon} \int_{\Omega}\left|u_{\varepsilon}-u\right|^{2} d x}\right) \\
& \times \sqrt{\frac{1}{\varepsilon} \int_{\Omega}\left|e^{i \psi_{\varepsilon}}-e^{i \varphi}\right|^{2} d x+\frac{1}{\varepsilon} \int_{\mathbb{R}^{2}}\left|\nabla \operatorname{div} \Delta^{-1}\left(\varphi_{n, \varepsilon}\right)\right|^{2} d x}
\end{aligned}
$$

Therefore, setting

$$
L_{0}:=\int_{J_{\varphi}}\left\{\int_{-\infty}^{+\infty}\left|\boldsymbol{v}(x) \cdot\left(e^{i \gamma(t, x)}-e^{i \varphi^{-}(x)}\right)\right|^{2} d t\right\} d \mathcal{H}^{1}(x),
$$

we have

$$
\begin{aligned}
\left|\frac{1}{\varepsilon} \int_{\mathbb{R}^{2}}\right| \nabla & \left.\operatorname{div} \Delta^{-1}\left(\chi_{\Omega} e^{i \psi_{\varepsilon}}\right)\right|^{2} d x-L_{0}|\leq| L_{0}-\frac{1}{\varepsilon} \int_{\mathbb{R}^{2}}\left|\nabla \operatorname{div} \Delta^{-1}\left(\varphi_{n, \varepsilon}\right)\right|^{2} d x \mid \\
& +4\left(\sqrt{\frac{1}{\varepsilon} \int_{\Omega}\left|e^{i \psi_{\varepsilon}}-\varphi_{n, \varepsilon}-u_{\varepsilon}\right|^{2} d x}+\sqrt{\frac{1}{\varepsilon} \int_{\Omega}\left|u_{\varepsilon}-u\right|^{2} d x}\right) \\
& \times \sqrt{\frac{1}{\varepsilon} \int_{\Omega}\left|e^{i \psi_{\varepsilon}}-e^{i \varphi}\right|^{2} d x+\frac{1}{\varepsilon} \int_{\mathbb{R}^{2}}\left|\nabla \operatorname{div} \Delta^{-1}\left(\varphi_{n, \varepsilon}\right)\right|^{2} d x}
\end{aligned}
$$

Using Proposition 2.2 with $W(a, b)=W\left(\left(a_{1}, a_{2}, a_{3}\right), b\right):\left(\mathbb{R} \times \mathbb{R}^{2} \times \mathbb{R}^{2}\right) \times \mathbb{R} \rightarrow$ $\mathbb{R}$, defined by $W(a, b):=\left|e^{i a_{1}}-a_{2}-a_{3}\right|^{2}$, we obtain,

$$
\begin{aligned}
& \lim _{\varepsilon \rightarrow 0} \frac{1}{\varepsilon} \int_{\Omega}\left|e^{i \psi_{\varepsilon}}-\varphi_{n, \varepsilon}-u_{\varepsilon}\right|^{2} d x=D_{n} \\
& :=\int_{J_{\varphi}}\left\{\int_{-\infty}^{+\infty}\left|e^{i \gamma(t, x)}-\left(\varphi^{+}(x)-\varphi^{-}(x)\right) \int_{t}^{+\infty} q_{n}(s, x) d s-\Gamma(t, x)\right|^{2} d t\right\} d \mathcal{H}^{1}(x),
\end{aligned}
$$

where $\gamma(t, x)$ is defined by (4.3), and

$$
\Gamma(t, x):=\left\{\int_{-\infty}^{t} \bar{p}(s, x) d s\right\} e^{i \varphi^{-}(x)}+\left\{\int_{t}^{+\infty} \bar{p}(s, x) d s\right\} e^{i \varphi^{+}(x)} .
$$


Using Proposition 2.2 with $W(a, b)=W: \mathbb{R}^{2} \times \mathbb{R}^{2} \rightarrow \mathbb{R}$, defined by $W(a, b):=$ $|a-b|^{2}$, we also infer that

$$
\begin{aligned}
& \lim _{\varepsilon \rightarrow 0} \frac{1}{\varepsilon} \int_{\Omega}\left|u_{\varepsilon}-u\right|^{2} d x=T(\bar{\eta}) \\
& :=\int_{J_{\varphi}}\left\{\int_{-\infty}^{0}\left|\Gamma(t, x)-u^{+}(x)\right|^{2} d t+\int_{0}^{+\infty}\left|\Gamma(t, x)-u^{-}(x)\right|^{2} d t\right\} d \mathcal{H}^{1}(x) \\
& =\int_{J_{\varphi}}\left\{\int_{-\infty}^{0}\left|\left(u^{+}-u^{-}\right) \int_{-\infty}^{t} \bar{p}(s, \cdot) d s\right|^{2} d t\right. \\
& \left.\quad+\int_{0}^{+\infty}\left|\left(u^{+}-u^{-}\right) \int_{t}^{+\infty} \bar{p}(s, \cdot) d s\right|^{2} d t\right\} d \mathcal{H}^{1},
\end{aligned}
$$

and

$$
\begin{aligned}
& \lim _{\varepsilon \rightarrow 0} \frac{1}{\varepsilon} \int_{\Omega}\left|e^{i \psi_{\varepsilon}}-e^{i \varphi}\right|^{2} d x \\
= & M:=\int_{J_{\varphi}}\left\{\int_{-\infty}^{0}\left|e^{i \gamma(t, x)}-e^{i \varphi^{+}(x)}\right|^{2} d t+\int_{0}^{+\infty}\left|e^{i \gamma(t, x)}-e^{i \varphi^{-}(x)}\right|^{2} d t\right\} d \mathcal{H}^{1}(x) .
\end{aligned}
$$

By Lemma 3.2 we obtain

$$
\begin{aligned}
& \lim _{\varepsilon \rightarrow 0} \int_{\mathbb{R}^{2}} \frac{1}{\varepsilon}\left|\nabla\left(\operatorname{div}\left(\Delta^{-1} \varphi_{n, \varepsilon}\right)\right)(x)\right|^{2} d x \\
& =L_{n}:=\int_{J_{\varphi}}\left\{\int_{-\infty}^{+\infty}\left|\varphi^{+}(x)-\varphi^{-}(x)\right|^{2} \cdot\left|\boldsymbol{v}(x) \cdot \int_{t}^{+\infty} q_{n}(s, x) d s\right|^{2} d t\right\} d \mathcal{H}^{1}(x) .
\end{aligned}
$$

Therefore, letting $\varepsilon$ tend to 0 in (4.18), we get,

$$
\begin{aligned}
\varlimsup_{\varepsilon \rightarrow 0^{+}} & \left.\left|\frac{1}{\varepsilon} \int_{\mathbb{R}^{2}}\right| \nabla \operatorname{div} \Delta^{-1}\left(\chi_{\Omega} e^{i \psi_{\varepsilon}}\right)\right|^{2} d x-L_{0} \mid \\
& \leq\left|L_{0}-L_{n}\right|+4\left(\sqrt{D_{n}}+\sqrt{T(\bar{\eta})}\right) \sqrt{M+L_{n}} .
\end{aligned}
$$

Using (4.7), (4.9), (4.13), (4.10) and (4.11) we obtain

$$
\lim _{n \rightarrow \infty} D_{n}=0,
$$


and since $\left(u^{+}(x)-u^{-}(x)\right) \perp \boldsymbol{v}(x)$ (by $\operatorname{div} u=0$ ), we also infer

$$
\lim _{n \rightarrow \infty} L_{n}=L_{0}:=\int_{J_{\varphi}}\left\{\int_{-\infty}^{+\infty}\left|\boldsymbol{v}(x) \cdot\left(e^{i \gamma(t, x)}-e^{i \varphi^{-}(x)}\right)\right|^{2} d t\right\} d \mathcal{H}^{1}(x) .
$$

Therefore, letting $n$ tend to $+\infty$ in (4.23), we obtain,

$$
\begin{aligned}
& \left.\varlimsup_{\varepsilon \rightarrow 0^{+}}\left|\frac{1}{\varepsilon} \int_{\mathbb{R}^{2}}\right| \nabla \operatorname{div} \Delta^{-1}\left(\chi_{\Omega} e^{i \psi_{\varepsilon}}\right)\right|^{2} d x-\iint_{J_{\varphi}}\left\{\int_{-\infty}^{+\infty}\left|\boldsymbol{v} \cdot\left(e^{i \gamma(t, \cdot)}-e^{i \varphi^{-}}\right)\right|^{2} d t\right\} d \mathcal{H}^{1} \mid \\
& \leq 4 \sqrt{T(\bar{\eta})} \sqrt{M+L_{0}} .
\end{aligned}
$$

This equation is valid for any $\bar{\eta} \in \mathcal{V}$, and the constants $M$ and $L_{0}$ do not depend on $\bar{\eta}$. For every $\delta>0$ we always can choose $\bar{\eta}_{\delta} \in C^{2}\left(\mathbb{R}^{2} \times \mathbb{R}^{2}, \mathbb{R}\right)$, satisfying $\bar{\eta}_{\delta} \geq 0$, supp $\bar{\eta}_{\delta} \Subset B_{\delta}(0) \times \mathbb{R}^{2}$ and $\int_{\mathbb{R}^{2}} \bar{\eta}_{\delta}(z, x) d z=1$ for any $x \in \Omega$. Then, as before, define $\bar{p}_{\delta}(t, x): \mathbb{R} \times J_{\varphi} \rightarrow \mathbb{R}$ by

$$
\bar{p}_{\delta}(t, x)=\int_{H_{\boldsymbol{v}(x)}^{0}} \bar{\eta}_{\delta}(t \boldsymbol{v}(x)+y, x) d \mathcal{H}^{1}(y) .
$$

Since $\bar{p}_{\delta} \geq 0$ and supp $\bar{p}_{\delta}(t, x) \subset[-\delta, \delta] \times J_{\varphi}$ and $\int_{-\infty}^{\infty} \bar{p}_{\delta}(t, x) d t=1$, by (4.20) we infer

$$
\begin{aligned}
& T\left(\bar{\eta}_{\delta}\right) \\
& \leq \int_{J_{\varphi}}\left\{\int_{-\delta}^{0}\left|\left(u^{+}-u^{-}\right) \int_{-\infty}^{t} \bar{p}_{\delta}(s, \cdot) d s\right|^{2} d t+\int_{0}^{\delta}\left|\left(u^{+}-u^{-}\right) \int_{t}^{+\infty} \bar{p}_{\delta}(s, \cdot) d s\right|^{2} d t\right\} d \mathcal{H}^{1} \\
& \leq 2 \delta \int_{J_{\varphi}}\left|u^{+}-u^{-}\right|^{2} d \mathcal{H}^{1} \leq 4 \delta \int_{J_{\varphi}}\left|u^{+}-u^{-}\right| d \mathcal{H}^{1} \leq 4 \delta\|D u\|(\Omega) .
\end{aligned}
$$

Therefore, by (4.26) we obtain

$$
\begin{aligned}
& \left.\varlimsup_{\varepsilon \rightarrow 0^{+}}\left|\frac{1}{\varepsilon} \int_{\mathbb{R}^{2}}\right| \nabla \operatorname{div} \Delta^{-1}\left(\chi_{\Omega} e^{i \psi_{\varepsilon}}\right)\right|^{2} d x-\iint_{J_{\varphi}}\left\{\int_{-\infty}^{+\infty}\left|\boldsymbol{v} \cdot\left(e^{i \gamma(t, \cdot)}-e^{i \varphi^{-}}\right)\right|^{2} d t\right\} d \mathcal{H}^{1} \mid \\
& \leq 8 \sqrt{\delta} \sqrt{\|D u\|(\Omega)} \sqrt{M+L_{0}} .
\end{aligned}
$$

For $\delta \rightarrow 0$, (4.27) gives (4.2). 
Let $\varphi, \bar{\varphi}$ and $\eta$ be as in Proposition 4.1 and $\psi_{\varepsilon}$ be defined by (4.1). Then using [16, Proposition 3.1], we obtain,

$$
\lim _{\varepsilon \rightarrow 0} \int_{\Omega} \varepsilon\left|\nabla \psi_{\varepsilon}(x)\right|^{2} d x=\int_{J_{\varphi}}\left|\varphi^{+}(x)-\varphi^{-}(x)\right|^{2} \cdot\left(\int_{\mathbb{R}} p^{2}(t, x) d t\right) d \mathcal{H}^{1}(x),
$$

where $p(t, x)$ is defined by (4.4). As in [16] and [17] we also easily deduce that

$$
\lim _{\varepsilon \rightarrow 0^{+}} \psi_{\varepsilon}(x)=\varphi(x) \quad \text { in } \quad L^{p}(\Omega, \mathbb{R}) \quad \forall p \in[1, \infty) .
$$

Combining these facts with the result of Proposition 4.1, we infer the following.

Corollary 4.2. Let $u \in B V\left(\Omega, S^{1}\right)$, satisfying $\operatorname{div} u=0$ in $\Omega$ and $u \cdot \boldsymbol{n}=0$ on $\partial \Omega$ ( $\boldsymbol{n}$ is the unit normal to $\partial \Omega$ ). Let $\varphi \in B V(\Omega, \mathbb{R}) \cap L^{\infty}$ such that $u=e^{i \varphi}$ a e. in $\Omega$. Consider a function $\bar{\varphi} \in B V\left(\mathbb{R}^{2}, \mathbb{R}\right) \cap L^{\infty}$ such that $\bar{\varphi}=\varphi$ a.e. in $\Omega$, supp $\bar{\varphi}$ is compact and $\|D \bar{\varphi}\|(\partial \Omega)=0$. Given $\eta \in \mathcal{V}$, for every $\varepsilon>0$ let $\psi_{\varepsilon}$ be defined by (4.1). Then,

$$
\begin{aligned}
& \lim _{\varepsilon \rightarrow 0}\left(\int_{\Omega} \varepsilon\left|\nabla e^{i \psi_{\varepsilon}(x)}\right|^{2} d x+\int_{\mathbb{R}^{2}}\left|\nabla \operatorname{div} \Delta^{-1}\left(\chi_{\Omega}(x) e^{i \psi_{\varepsilon}(x)}\right)\right|^{2} d x\right) \\
& =Y_{\varphi}(\eta):=\int_{J_{\varphi}}\left|\varphi^{+}(x)-\varphi^{-}(x)\right|^{2} \cdot\left(\int_{-\infty}^{+\infty} p^{2}(t, x) d t\right) d \mathcal{H}^{1}(x) \\
& \quad+\int_{J_{\varphi}}\left\{\int_{-\infty}^{+\infty}\left|\boldsymbol{v}(x) \cdot\left(e^{i \gamma(t, x)}-e^{i \varphi^{-}(x)}\right)\right|^{2} d t\right\} d \mathcal{H}^{1}(x),
\end{aligned}
$$

where $\gamma$ and $p$ defined by (4.3) and (4.4) respectively. Moreover,

$$
\lim _{\varepsilon \rightarrow 0^{+}} \psi_{\varepsilon}(x)=\varphi(x) \quad \text { in } \quad L^{p}(\Omega, \mathbb{R}) \quad \forall p \in[1, \infty) .
$$

Next we turn to the minimization problem of the term on the right hand side of (4.29), over all kernels $\eta \in \mathcal{V}$, analogously to that was done in [16] and [17]. By the same method, as there, we can obtain the following.

Lemma 4.3. Let $Y_{\varphi}(\eta): \mathcal{V} \rightarrow \mathbb{R}$ be defined as the right hand side of (4.29). Then,

$$
\begin{aligned}
& \inf _{\eta \in \mathcal{V}} Y_{\varphi}(\eta)=\mathcal{J}_{0}(\varphi) \\
& :=\int_{J_{\varphi}} 2\left|\varphi^{+}(x)-\varphi^{-}(x)\right|\left\{\int_{0}^{1}\left|\boldsymbol{v}(x) \cdot\left(e^{i\left(s \varphi^{-}(x)+(1-s) \varphi^{+}(x)\right)}-e^{i \varphi^{-}(x)}\right)\right| d s\right\} d \mathcal{H}^{1}(x) \\
& =\int_{J_{\varphi}} 2\left|\int_{\varphi^{-}(x)}^{\varphi^{+}(x)}\right| \boldsymbol{v}(x) \cdot\left(e^{i t}-e^{i \varphi^{-}(x)}\right)|d t| d \mathcal{H}^{1}(x) .
\end{aligned}
$$


By [19, (II.36)] we infer that

$$
\begin{aligned}
\mathcal{J}_{0}(\varphi) & =\int_{J_{\varphi}} 2\left|\int_{\varphi^{-}(x)}^{\varphi^{+}(x)}\right| \boldsymbol{v}(x) \cdot\left(e^{i t}-e^{i \varphi^{-}(x)}\right)|d t| d \mathcal{H}^{1}(x) \\
& =2 \int_{\mathbb{R}} \int_{\Omega}\left|\operatorname{div}_{x} T^{t} u\right| d x d t
\end{aligned}
$$

where we (as in [19]), consider $T^{t} \varphi:=\inf (\varphi, t)$ and $T^{t} u:=e^{i T^{t} \varphi}$.

Proof of Theorem 1.2. The case of $\varphi \in B V(\Omega, \mathbb{R}) \cap L^{\infty}$ follows easily from Corollary 4.2 and Lemma 4.3 by using a standard diagonal argument as in the proofs of [17, Theorem 1.1 and Theorem 1.2].

It remains to consider the case of an unbounded $\varphi \in B V(\Omega, \mathbb{R})$, such that $e^{i \varphi(x)}=u(x)$ a.e. in $\Omega$. First recall that by [6] there exists $\varphi_{0} \in B V(\Omega, \mathbb{R}) \cap$ $L^{\infty}(\Omega, \mathbb{R})$ satisfying $e^{i \varphi_{0}(x)}=u(x)$ a.e. in $\Omega$. Then $\varphi(x)=\varphi_{0}(x)+2 \pi l(x)$ where $l \in B V(\Omega, \mathbb{Z})$. For each integer $n \geq 1$ define,

$$
l_{n}(x):=\left\{\begin{array}{ll}
l(x) & x \in \Omega,|l(x)| \leq n, \\
n & x \in \Omega, l(x)>n, \\
-n & x \in \Omega, l(x)<-n,
\end{array} \quad \varphi_{n}(x):=\varphi_{0}(x)+2 \pi l_{n}(x) .\right.
$$

Clearly $\varphi_{n} \in B V(\Omega) \cap L^{\infty}(\Omega)$ and $e^{i \varphi_{n}(x)}=u(x)$ a.e. in $\Omega$. From the case of a bounded $\varphi$, considered above, it follows that for each $n$ there exists a family $\left\{v_{n, \varepsilon}\right\}_{\varepsilon>0} \subset C^{2}(\Omega, \mathbb{R})$ satisfying $\lim _{\varepsilon \rightarrow 0} v_{n, \varepsilon}(x)=\varphi_{n}(x)$ in $L^{1}(\Omega, \mathbb{R})$ and

$$
\begin{aligned}
& \lim _{\varepsilon \rightarrow 0}\left(\int_{\Omega} \varepsilon\left|\nabla e^{i v_{n, \varepsilon}(x)}\right|^{2} d x+\int_{\mathbb{R}^{2}}\left|\nabla \operatorname{div} \Delta^{-1}\left(\chi_{\Omega}(x) e^{i v_{n, \varepsilon}(x)}\right)\right|^{2} d x\right) \\
& =\mathcal{J}_{0}\left(\varphi_{n}\right)=\int_{J_{\varphi_{n}}} 2\left|\int_{\varphi_{n}^{-}(x)}^{\varphi_{n}^{+}(x)}\right| \boldsymbol{v}_{n}(x) \cdot\left(e^{i t}-e^{i \varphi_{n}^{-}(x)}\right)|d t| d \mathcal{H}^{1}(x)
\end{aligned}
$$

Since for any $x \in \Omega$ we have $\left|\varphi_{n}(x)\right| \leq\left|\varphi_{0}(x)\right|+2 \pi|l(x)|$ while $\varphi_{n}(x)=\varphi(x)$ for $n$ sufficiently large, we deduce by dominated convergence that

$$
\lim _{n \rightarrow \infty} \varphi_{n}(x)=\varphi(x) \quad \text { in } L^{1}(\Omega, \mathbb{R}) .
$$

Put $\lambda_{n}(x):=\left|\varphi_{n}^{+}(x)-\varphi_{n}^{-}(x)\right|$. For $\mathcal{H}^{N-1}$-almost every $x \in J_{\varphi_{0}} \cup J_{l}$ we have $\lambda_{n}(x) \leq\left|\varphi_{0}^{+}(x)-\varphi_{0}^{-}(x)\right|+2 \pi\left|l^{+}(x)-l^{-}(x)\right|$, while $\lambda_{n}(x)=\left|\varphi^{+}(x)-\varphi^{-}(x)\right|$ for sufficiently large $n$. Moreover, $\mathcal{H}^{N-1}\left(J_{\varphi_{n}} \backslash\left(J_{\varphi_{0}} \cup J_{l}\right)\right)=0$ and $\boldsymbol{v}_{n}(x)=\boldsymbol{v}(x)$ 
for $\mathcal{H}^{N-1}$-a.e. $x \in J_{\varphi_{n}} \cap J_{\varphi}$, for each $n$. Therefore, by dominated convergence,

$$
\begin{aligned}
& \lim _{n \rightarrow \infty} \int_{J_{\varphi_{n}}} 2\left|\int_{\varphi_{n}^{-}(x)}^{\varphi_{n}^{+}(x)}\right| \boldsymbol{v}_{n}(x) \cdot\left(e^{i t}-e^{i \varphi_{n}^{-}(x)}\right)|d t| d \mathcal{H}^{1}(x) \\
& =\int_{J_{\varphi}} 2\left|\int_{\varphi^{-}(x)}^{\varphi^{+}(x)}\right| \boldsymbol{v}(x) \cdot\left(e^{i t}-e^{i \varphi^{-}(x)}\right)|d t| d \mathcal{H}^{1}(x)
\end{aligned}
$$

To complete the proof, we apply to $\left\{v_{n, \varepsilon}\right\}$ a standard diagonal argument.

\section{References}

[1] L. Ambrosio, C. De Lellis and C. Mantegazza, Line energies for gradient vector fields in the plane, Calc. Var. Partial Differential Equations 9 (1999), 327-355.

[2] L. Ambrosio, N. Fusco and D. Pallara, "Functions of Bounded Variation and Free Discontinuity Problems", Oxford Mathematical Monographs, Oxford University Press, New York, 2000.

[3] P. Aviles and Y. GigA, A mathematical problem related to the physical theory of liquid crystal configurations, Proc. Centre Math. Anal. Austral. Nat. Univ. 12 (1987), 1-16.

[4] P. AVILES and Y. GIGA, On lower semicontinuity of a defect energy obtained by a singular limit of the Ginzburg-Landau type energy for gradient fields, Proc. Roy. Soc. Edinburgh Sect. A 129 (1999), 1-17.

[5] S. ConTI and C. DE LELLIS, Sharp upper bounds for a variational problem with singular perturbation, Math. Ann. 338 (2007), 119-146.

[6] J. DÁvila and R. IGnAt, Lifting of $B V$ functions with values in $S^{1}$, C. R. Math. Acad. Sci. Paris 337 (2003) 159-164.

[7] C. DE LELLIS, An example in the gradient theory of phase transitions ESAIM Control Optim. Calc. Var. 7 (2002), 285-289 (electronic).

[8] A. DeSimone, S. MÜller, R. V. Kohn and F. Otto, A compactness result in the gradient theory of phase transitions, Proc. Roy. Soc. Edinburgh Sect. A 131 (2001), 833-844.

[9] N. M. ERCOLAni, R. INDiK, A. C. NeWEll and T. PASSOT, The geometry of the phase diffusion equation, J. Nonlinear Sci. 10 (2000), 223-274.

[10] L. C. Evans, "Partial Differential Equations", Graduate Studies in Mathematics, Vol. 19, American Mathematical Society, 1998.

[11] L. C. Evans and R. F. GARIEPy, "Measure Theory and Fine Properties of Functions", Studies in Advanced Mathematics, CRC Press, Boca Raton, FL, 1992.

[12] D. Gilbarg and N. Trudinger, "Elliptic Partial Differential Equations of Elliptic Type", 2nd ed., Springer-Verlag, Berlin-Heidelberg, 1983.

[13] E. GIUSTI, "Minimal Surfaces and Functions of Bounded Variation", Monographs in Mathematics, Vol. 80, Birkhäuser Verlag, Basel, 1984.

[14] W. JIN and R.V. KoHN, Singular perturbation and the energy of folds, J. Nonlinear Sci. 10 (2000), 355-390.

[15] A. POLIAKOVSKY, A method for establishing upper bounds for singular perturbation problems, C. R. Math. Acad. Sci. Paris 341 (2005), 97-102.

[16] A. POLIAKOVSKY, Upper bounds for singular perturbation problems involving gradient fields, J. Eur. Math. Soc. 9 (2007), 1-43.

[17] A. Poliakovsky, A general technique to prove upper bounds for singular perturbation problems, submitted to Journal d'Analyse. 
[18] T. RIVIÈRE and S. SERFATY, Limiting domain wall energy for a problem related to micromagnetics, Comm. Pure Appl. Math. 54 (2001), 294-338.

[19] T. RIVIÈRE and S. SERFATY, Compactness, kinetic formulation and entropies for a problem related to mocromagnetics, Comm. Partial Differential Equations 28 (2003), 249-269.

[20] A. I. Volpert and S. I. Hudjaev, "Analysis in Classes of Discontinuous Functions and Equations of Mathematical Physics", Martinus Nijhoff Publishers, Dordrecht, 1985.

Institut für Mathematik

Universität Zürich

Winterthurerstrasse 190

CH-8057 Zürich, Switzerland

apoliako@math.unizh.ch 